\title{
Total 4-choosability of series-parallel graphs
}

\author{
Douglas R. Woodall \\ School of Mathematical Sciences \\ University of Nottingham \\ Nottingham NG7 2RD, UK \\ douglas.woodall@nottingham.ac.uk
}

Submitted: Jan 25, 2005; Accepted: Oct 18, 2006; Published: Oct 31, 2006

Mathematics Subject Classification: 05C15

\begin{abstract}
It is proved that, if $G$ is a $K_{4}$-minor-free graph with maximum degree 3 , then $G$ is totally 4-choosable; that is, if every element (vertex or edge) of $G$ is assigned a list of 4 colours, then every element can be coloured with a colour from its own list in such a way that every two adjacent or incident elements are coloured with different colours. Together with other known results, this shows that the List-Total-Colouring Conjecture, that $\operatorname{ch}^{\prime \prime}(G)=\chi^{\prime \prime}(G)$ for every graph $G$, is true for all $K_{4}$-minor-free graphs and, therefore, for all outerplanar graphs.
\end{abstract}

Keywords: Outerplanar graph; Minor-free graph; Series-parallel graph; List total colouring.

\section{Introduction}

We use standard terminology, as defined in the references: for example, [8] or [10]. We distinguish graphs (which are always simple) from multigraphs (which may have multiple edges); however, our theorem is only for graphs. For a graph (or multigraph) $G$, its edge chromatic number, total (vertex-edge) chromatic number, edge choosability (or list edge chromatic number), total choosability, and maximum degree, are denoted by $\chi^{\prime}(G)$, $\chi^{\prime \prime}(G), \operatorname{ch}^{\prime}(G), \operatorname{ch}^{\prime \prime}(G)$, and $\Delta(G)$, respectively. So $\operatorname{ch}^{\prime \prime}(G)$ is the smallest $k$ for which $G$ is totally $k$-choosable.

There is great interest in discovering classes of graphs $G$ for which the choosability or list chromatic number $\operatorname{ch}(G)$ is equal to the chromatic number $\chi(G)$. The List-EdgeColouring Conjecture (LECC) and List-Total-Colouring Conjecture (LTCC) $[1,4,6]$ are that, for every multigraph $H, \operatorname{ch}^{\prime}(H)=\chi^{\prime}(H)$ and $\operatorname{ch}^{\prime \prime}(H)=\chi^{\prime \prime}(H)$, respectively; so the conjectures are that $\operatorname{ch}(G)=\chi(G)$ whenever $G$ is the line graph or the total graph of a multigraph $H$. 
For an outerplanar (simple) graph $H$, Wang and Lih [9] proved that $\operatorname{ch}^{\prime}(H)=\chi^{\prime}(H)=$ $\Delta(H)$ if $\Delta(H) \geqslant 3$ and $\operatorname{ch}^{\prime \prime}(H)=\chi^{\prime \prime}(H)=\Delta(H)+1$ if $\Delta(H) \geqslant 4$. For the larger class of $K_{4}$-minor-free (series-parallel) graphs, the first of these results had already been proved by Juvan, Mohar and Thomas [7], and the second was proved by Hetherington and Woodall [5], following an incomplete outline proof by Zhou, Matsuo and Nishizeki [12]. This proves both the LECC and the LTCC for $K_{4}$-minor-free (simple) graphs, except for the following missing case, the proof of which is the sole achievement of this paper.

Theorem 1. If $H$ is a $K_{4}$-minor-free graph with maximum degree 3 , then $\operatorname{ch}^{\prime \prime}(H)=$ $\chi^{\prime \prime}(H)=4$.

In Section 2 we set up the framework for proving Theorem 1, and prove it subject to a number of technical lemmas; these lemmas are proved in Sections 3-5. The resulting proof of Theorem 1 is very long; it would clearly be desirable to have a shorter proof.

For brevity, when considering total colourings of a graph $G$, we will sometimes say that a vertex and an edge incident to it are adjacent or neighbours, since they correspond to adjacent or neighbouring vertices of the total graph $T(G)$ of $G$. In the context of this paper, by a 4-list-assignment $\Lambda$ to a graph $G$ we always mean an assignment of a list $\Lambda(z)$ of four colours to every element (vertex or edge) $z$ of $G$.

\section{The framework for the proof}

We first define the concept of a sepachain (short for series-parallel chain). Consider first a graph $G$ containing exactly two vertices $u, v$ with degree 1 , with neighbours $x, y$ respectively. It is convenient to draw $G$ as in Fig. 1(a); note however that $u$ and $v$ are vertices of $G$, despite being outside the region labelled $G$ in the figure. The sepachains form a subclass of graphs of this type. They are defined inductively as follows: a path (with at least one edge) is a sepachain; and if $G_{1}$ and $G_{2}$ are sepachains then the graphs formed by joining them in series and in parallel, as in Figs 1(b) and 1(d), are both sepachains. A sepachain is nontrivial if it is not a path of length 1 or 2 ; that is, if the vertices $u, x, y, v$ in Fig. 1(a) are all distinct. The relevance of sepachains is shown by the following easy lemma.

Lemma 1. Let $H$ be a $K_{4}$-minor-free block with maximum degree at most 3. Suppose $H$ contains a vertex $z_{0}$ of degree 2 , with neighbours $x, y$, and $H^{\prime}$ is formed from $H$ by replacing $z_{0}$ by two vertices $u, v$ of degree 1 with neighbours $x, y$ respectively. Then $H^{\prime}$ is a nontrivial sepachain.

Proof. We prove the result by induction on $|V(H)|$. It is clear that $x \neq y$, so that $H^{\prime}$ is not a trivial sepachain. The result holds if $H^{\prime}$ is a path, so suppose that it is not.

Suppose first that there do not exist two edge-disjoint $x y$-paths in $H^{\prime}$. Then, by the edge-separation analogue of Menger's theorem, there is a cutedge in $H^{\prime}$ separating $x$ from $y$; that is, $H^{\prime}$ can be labelled as in Fig. 1(b), where $y_{1} v_{1}=u_{2} x_{2}$ is the cutedge. For $i=1,2$, 


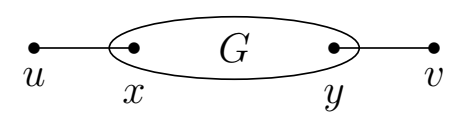

(a)

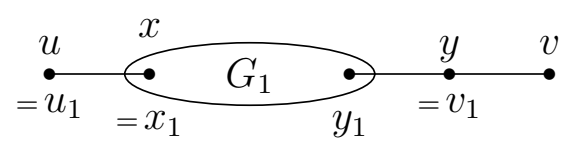

(c)

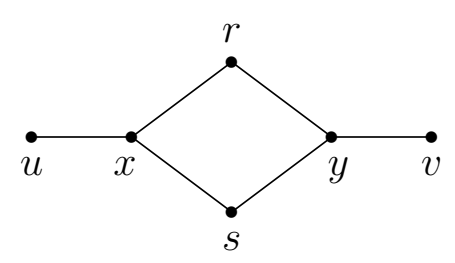

(e)

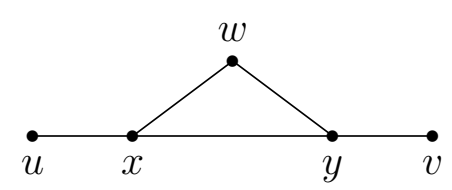

(g)

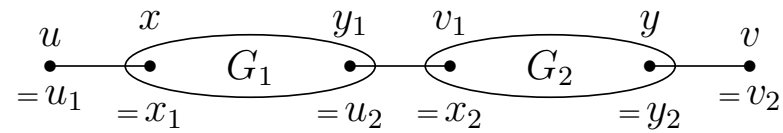

(b)

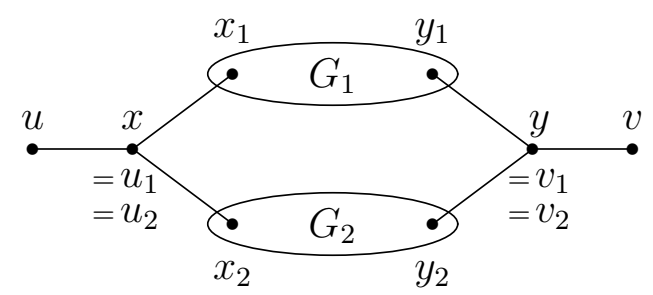

(d)

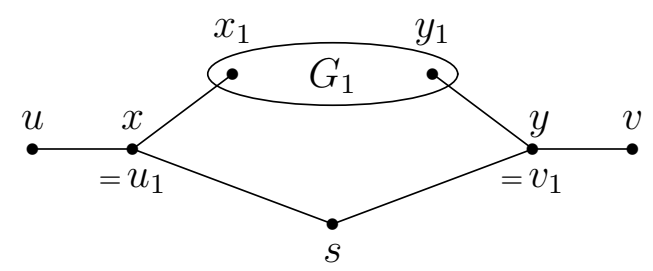

(f)

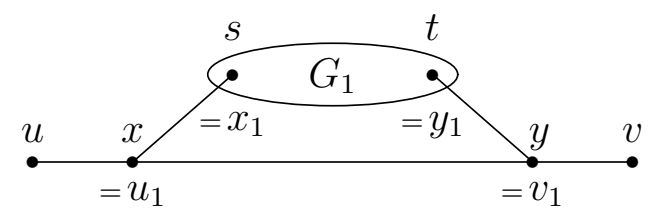

(h)

Fig. 1 


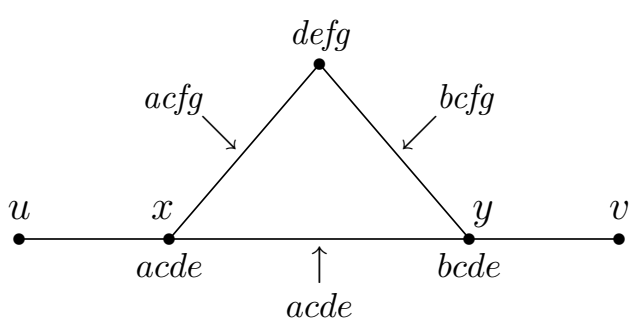

(a)

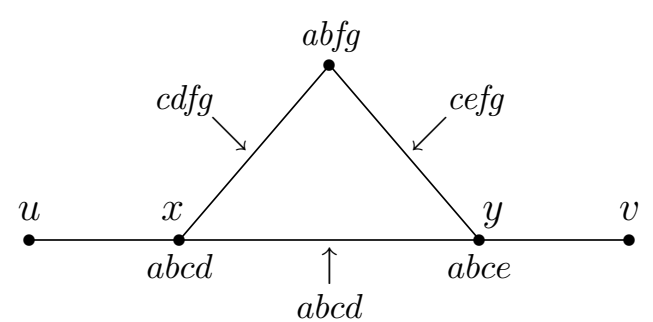

(b)

Fig. 2

if $x_{i}=y_{i}$ then $G_{i}$ is a path of length 2 , which is a (trivial) sepachain, and if $x_{i} \neq y_{i}$ then we may suppose that $G_{i}$ is a (nontrivial) sepachain by the induction hypothesis applied to the $K_{4}$-minor-free block obtained from $G_{i}$ by identifying $u_{i}$ with $v_{i}$. Thus $H^{\prime}$ is a sepachain, formed by joining $G_{1}$ and $G_{2}$ in series.

Suppose now that there exist two edge-disjoint $x y$-paths in $H^{\prime}$, necessarily internally vertex-disjoint and with no connection between them, since $H$ has maximum degree at most 3 and no $K_{4}$ minor. If one of these paths is a single edge, then $H^{\prime}$ looks like Fig. 1(h), where if $G_{1}$ is not a path of length 2 (as in Fig. 1(g)) then it is a nontrivial sepachain, by the induction hypothesis applied to the $K_{4}$-minor-free block obtained from $G_{1}$ by identifying $u_{1}$ with $v_{1}$. If neither of the paths is a single edge, then $H^{\prime}$ looks like Fig. 1(d), where each of $G_{1}, G_{2}$ is either a path of length 2 (as in Fig. 1(e) or 1(f)) or a nontrivial sepachain, by the same inductive argument. In all cases, $H^{\prime}$ is a nontrivial sepachain, formed by joining $G_{1}$ and $G_{2}$ in parallel.

We say that a nontrivial sepachain $G$ (labelled as in Fig. 1(a)) is very good for total 4-choosability if, for each 4-list-assignment $\Lambda$ to $G$, and each way of colouring the elements $u, u x, v y, v$ with colours $\lambda_{u}, \lambda_{u x}, \lambda_{v y}, \lambda_{v}$ from their lists, this colouring can be extended to a total $\Lambda$-colouring of $G$. Not every sepachain is very good for total 4-choosability, since if $x, y$ are adjacent, and every element is given list $\{a, b, c, d\}$, and $u, u x, v y, v$ are coloured with colours $c, d, c, d$ respectively, then this colouring cannot be extended to the elements $x, x y, y$ (which must all be given different colours but can only be coloured with $a$ or $b$ ). Because of this and similar examples, we introduce a weaker concept.

We say that a nontrivial sepachain $G$ is good for total 4-choosability if, for each 4-listassignment $\Lambda$ to $G$, there is one of the conditions in Table 1 such that, if the elements $u, u x, v y, v$ are given colours $\lambda_{u}, \lambda_{u x}, \lambda_{v y}, \lambda_{v}$ from their lists in a way that does not match any of the forbidden patterns given for that condition in the table (and $\lambda_{u} \neq \lambda_{u x}$ and $\lambda_{v y} \neq \lambda_{v}$, of course), then this colouring can be extended to a total $\Lambda$-colouring of $G$. Here $a, b, c, d, e, f$ are distinct specific colours (depending on $G$ and $\Lambda$ ), $\mu, \nu, \xi$ are variable colours, and a dot denotes an arbitrary colour (so that, for example, $\cdot \xi \xi \cdot$ denotes any colouring in which $u x$ and $v y$ are given the same colour). For example, if the the pair $(G, \Lambda)$ is as in Fig. 2(a), where the lists are written as abcd rather than $\{a, b, c, d\}$, and 


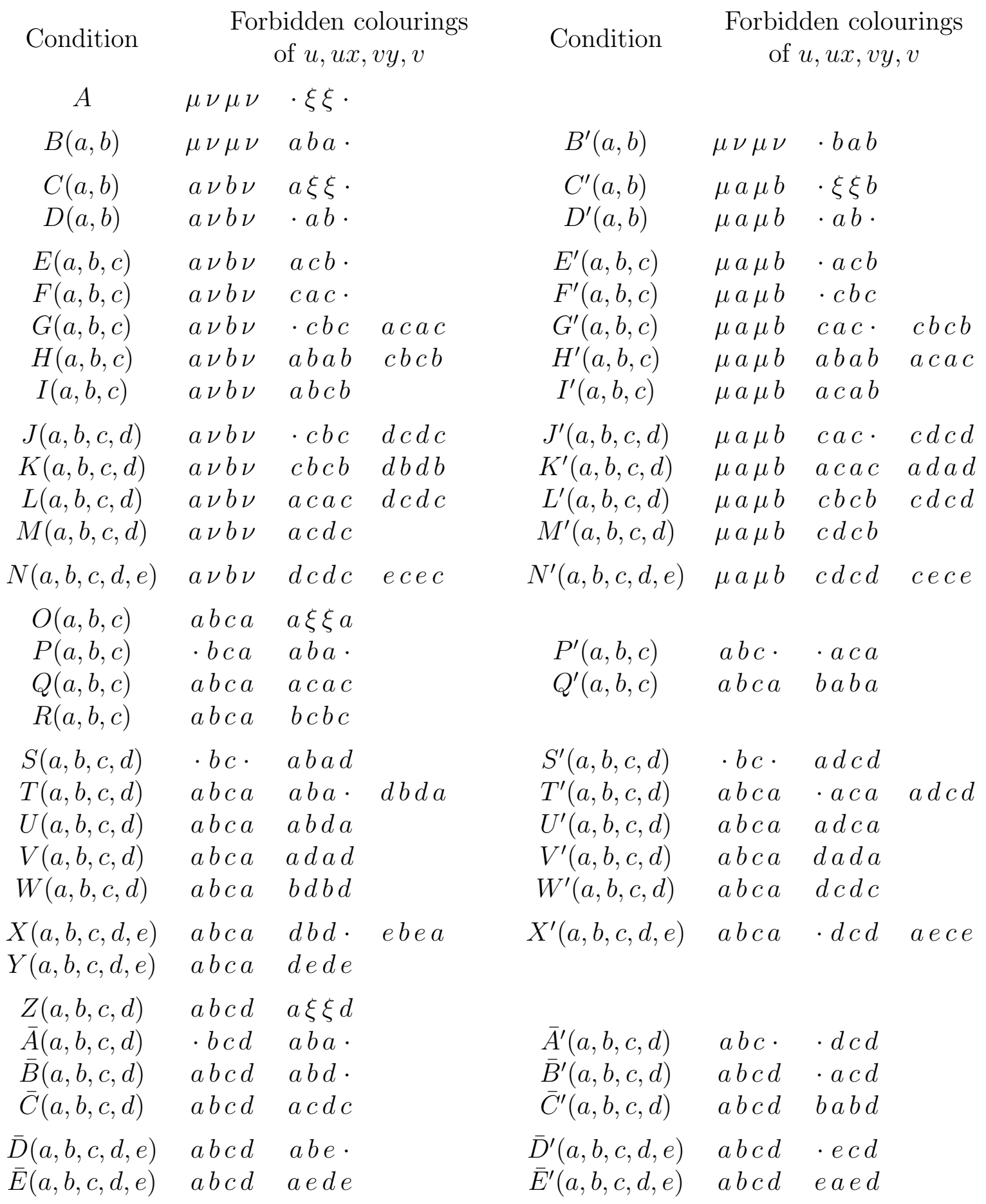

Table 1 
if the elements $u, u x, v y, v$ are given colours $\lambda_{u}, \lambda_{u x}, \lambda_{v y}, \lambda_{v}$, then this colouring can be extended to a total $\Lambda$-colouring of $G$ unless $\lambda_{u}=\lambda_{v y} \in\{c, d, e\}$ and $\left(\lambda_{u x}, \lambda_{v}\right)=(a, b)$, or $\left(\lambda_{u}, \lambda_{u x}, \lambda_{v y}, \lambda_{v}\right)=(c, a, b, c)$; hence it can be extended if $\lambda_{u}, \lambda_{u x}, \lambda_{v y}, \lambda_{v}$ does not match either of the patterns given for condition $D^{\prime}(a, b)$ in Table 1 . The precise form of Table 1 is determined by the proof of Lemma 9 .

We say that a colouring of $u, u x, v y, v$ satisfies condition $\Xi$ if it does not match any of the patterns given for condition $\Xi$ in Table 1 . A pair $(G, \Lambda)$, comprising a sepachain $G$ with associated 4-list-assignment $\Lambda$, is of type $\Xi$ if every colouring that satisfies condition $\Xi$ can be extended to a total $\Lambda$-colouring of $G$, but this is not true for any earlier condition in Table 1. According to this definition, $(G, \Lambda)$ cannot have more than one type, and Theorem 2 implies that if $G$ is nontrivial then $(G, \Lambda)$ has exactly one type. For example, if $(G, \Lambda)$ is as in Fig. 2(a) then it has type $D^{\prime}(a, b)$; if it is as in Fig. 2(b) (which is the same with a different labelling of the colours) then it has type $D^{\prime}(d, e)$; and if $G$ is very good for total 4-choosability then $(G, \Lambda)$ has type $A$, for every 4-list-assignment $\Lambda$.

Our proof of Theorem 1 rests on the following result.

Theorem 2. Every nontrivial sepachain $G$ is good for total 4-choosability.

Outline proof. We prove the result by induction on $|V(G)|$. The base case for the induction is a path of length 3 , whose goodness follows from that of the configuration in Fig. 1(g), proved in Lemma 8. If $G$ is a nontrivial sepachain that is not a path of length 3, then it is formed by joining two smaller, possibly trivial, sepachains $G_{1}$ and $G_{2}$ in series or in parallel.

If $G$ is formed by joining $G_{1}$ and $G_{2}$ in series, then we may assume that neither $G_{1}$ nor $G_{2}$ is a single edge (since if $G_{2}$, say, is a single edge, then $G \cong G_{1}$ ), and that $G_{1}$ and $G_{2}$ are not both paths of length 2 (since, if they are, then $G$ is a path of length 3 ). We may assume inductively that if $G_{1}$ or $G_{2}$ is nontrivial then it is good. If $G_{2}$, say, is a path of length 2, then $G$ is as in Fig. 1(c), and it is proved good in Lemma 2. If $G_{1}$ and $G_{2}$ are both nontrivial then $G$ is as in Fig. 1(b), and it is proved good in Lemma 3.

If $G$ is formed by joining $G_{1}$ and $G_{2}$ in parallel, then each of $G_{1}$ and $G_{2}$ can be a single edge, a path of two edges, or nontrivial, except that $G_{1}$ and $G_{2}$ cannot both be single edges since $G$ is simple. If $G_{2}$, say, is a single edge, then $G$ is as in Fig. 1(g) or $1(\mathrm{~h})$, and it is proved good in Lemma 8 or 9 respectively. If $G_{2}$ is a path of length 2 and $G_{1}$ is not a single edge, then $G$ is as in Fig. 1(e) or 1(f), and it is proved good in Lemma 7 or 6 respectively. Finally, if $G_{1}$ and $G_{2}$ are both nontrivial then $G$ is as in Fig. 1(d), and it is proved good in Lemma 5.

We now show how Theorem 2 implies Theorem 1.

Proof of Theorem 1. Let $H$ be a $K_{4}$-minor-free graph with maximum degree 3 . It is clear that $\operatorname{ch}^{\prime \prime}(H) \geqslant \chi^{\prime \prime}(H) \geqslant 4$, and so it suffices to prove that $\operatorname{ch}^{\prime \prime}(H) \leqslant 4$. In proving this we assume only that the maximum degree of $H$ is at most 3. Suppose if possible that $\operatorname{ch}^{\prime \prime}(H)>4$ and that $H$ has as few vertices as possible subject to this condition. It is clear that $H$ is connected and has no vertex with degree 0 or 1. 
If $H$ is 2-connected let $B=H$ and let $z_{0}$ be any vertex of degree 2 in $H$, which exists by the well-known result of Dirac [2] that every $K_{4}$-minor-free graph has a vertex with degree at most 2. If $H$ is not 2-connected let $B$ be an end-block of $H$ with cutvertex $z_{0}$. In either case, $z_{0}$ has degree 2 in $B$. Let its neighbours in $B$ be $x$ and $y$, and let $G$ be the graph obtained from $B$ by replacing $z_{0}$ by two vertices $u, v$ of degree 1 with neighbours $x, y$ respectively. By Lemma $1, G$ is a nontrivial sepachain.

Let $\Lambda$ be a 4 -list-assignment to $H$ such that $H$ has no total $\Lambda$-colouring. If $B \neq H$ then we may suppose that $H-\left(B-z_{0}\right)$ has a total $\Lambda$-colouring; uncolour $z_{0}$, and for each uncoloured element $z$ let $L(z)$ denote the residual list of colours in $\Lambda(z)$ that are not used on any element adjacent to $z$, and so are still available for use on $z$. If $B=H$ let $L(z)=\Lambda(z)$ for every element $z$. In either case,

$$
|L(z)| \geqslant \begin{cases}2 & \text { if } z=z_{0} \\ 3 & \text { if } z=z_{0} x \text { or } z_{0} y \\ 4 & \text { otherwise }\end{cases}
$$

We can transfer these lists to $G$ by defining $L(u)=L(v)=L\left(z_{0}\right), L(u x)=L\left(z_{0} x\right)$ and $L(v y)=L\left(z_{0} y\right)$. A total $L$-colouring of $H$ corresponds to a total $L$-colouring of $G$ in which $u, v$ have the same colour and $u, u x, v y$ have three different colours. A study of Table 1 shows that for every type of $G$, we can ensure that a colouring of $u, u x, v y, v$ with these properties can be extended to a total $L$-colouring of $G$ provided that we avoid a fixed colour on one of $u x$ and $v y$. Specifically, if $G$ has type $D(a, b), D^{\prime}(a, b)$ or $E^{\prime}(a, b, c)$, then such a colouring will extend provided that $u x$ does not have colour $a$. If $G$ has type $E(a, b, c)$, then it is enough that $u x$ does not have colour $c$. If $G$ has one of types $O-Y$, $\bar{A}(a, b, c, d), \bar{B}(a, b, c, d)$ or $\bar{D}(a, b, c, d, e)$, then it is enough that $u x$ does not have colour $b$. If $G$ has one of types $P^{\prime}-X^{\prime}, \bar{A}^{\prime}(a, b, c, d), \bar{B}^{\prime}(a, b, c, d)$ or $\bar{D}^{\prime}(a, b, c, d, e)$, then it is enough that $v y$ does not have colour $c$. If $G$ has any other type, then no restriction is needed.

If we must avoid a particular colour $a$ on $u x$, then colour $u x$ (and $z_{0} x$ ) first with a colour $b \in L\left(z_{0} x\right) \backslash\{a\}$, then colour $u$ (and $v$ and $z_{0}$ ) with a colour $c \in L\left(z_{0}\right) \backslash\{b\}$, and finally colour $v y$ (and $z_{0} y$ ) with a colour $d \in L\left(z_{0} y\right) \backslash\{b, c\}$. If we must avoid a particular colour on $v y$, then colour $v y, u, u x$ in the reverse order. Either way, this colouring extends to a total $L$-colouring of $G$ and hence to a total $\Lambda$-colouring of $H$, and this contradiction completes the proof of Theorem 1.

The remainder of the paper is devoted to the lemmas needed to prove Theorem 2.

\section{The series constructions}

In this section we will use only the following property of good sepachains, which can be seen by a careful study of Table 1: if $\Lambda$ is a 4-list-assignment to a nontrivial good sepachain $G$, and $u$ and $u x$ are coloured from their lists, and we wish to extend this colouring to a total $\Lambda$-colouring of $G$, then there are at most two possible choices for the colour of $v y$ that place any restriction at all on the colour of $v$, and there is at most one choice that 
forbids more than one colour for $v$ (in addition to the obvious restriction that $v$ must have a different colour from $v y$ ).

Lemma 2. If a nontrivial sepachain $G_{1}$ is good for total 4-choosability, then the sepachain $G$ in Fig. 1(c) is very good for total 4-choosability.

Proof. Let $\Lambda$ be a 4-list-assignment to $G$, and let colours $\lambda_{u}, \lambda_{u x}, \lambda_{v y}, \lambda_{v}$ be assigned to $u, u x, v y, v$. At this point there are at least two colours that we can use on the vertex $v_{1}=y$ and at least three colours that we can use on the edge $v_{1} y_{1}$. So give $v_{1} y_{1}$ a colour that places no restriction at all on the colour of $v_{1}$ (if we want to extend this colouring to $G_{1}$ ), and then give $v_{1}$ a colour different from the colour we have just given to $v_{1} y_{1}$. Now this colouring of $u=u_{1}, u x=u_{1} x_{1}, v_{1} y_{1}$ and $v_{1}$ can be extended to the whole of $G_{1}$, and so the original colouring of $u, u x, v y, v$ can be extended to a total $\Lambda$-colouring of $G$.

Lemma 3. If $G_{1}$ and $G_{2}$ are nontrivial sepachains that are good for total 4-choosability, then the sepachain $G$ in Fig. 1(b) is very good for total 4-choosability.

Proof. Let $\Lambda$ be a 4-list-assignment to $G$, and let colours $\lambda_{u}, \lambda_{u x}, \lambda_{v y}, \lambda_{v}$ be assigned to $u, u x, v y, v$. Consider the edge $y_{1} v_{1}=u_{2} x_{2}$. There are at most two of the four possible colours for this edge that place any restriction at all on the colour of $u_{2}$ (if we want to extend this colouring to $G_{2}$ ), and there is at most one colour for this edge that forbids more than one colour for $v_{1}$ (if we want to extend this colouring to $G_{1}$ ). So give this edge a colour that places no restriction on $u_{2}$ and forbids at most one colour for $v_{1}$, and give $v_{1}$ a colour that is not forbidden (and is different from the colour of the edge). Now this colouring can be extended to the whole of $G_{1}$. If we now delete the colour that we assigned to $v_{1}$, then the resulting colouring of $u_{2}, u_{2} x_{2}, v_{2} y_{2}=v y$ and $v_{2}=v$ can be extended to the whole of $G_{2}$. Thus the original colouring of $u, u x, v y, v$ can be extended to a total $\Lambda$-colouring of $G$.

\section{The easier parallel constructions}

In this section we will need the following easy lemma.

Lemma 4. (a) $\operatorname{ch}^{\prime}\left(C_{4}\right)=\chi^{\prime}\left(C_{4}\right)=2$.

(b) If $C: x w_{1} y w_{2} x$ is a 4-cycle and every edge $z$ of $C$ is given a list $\Gamma(z)$ of three colours, and if $\mu, \nu$ are arbitrary colours, then the edges of $C$ can be coloured from their lists in such a way that adjacent edges get different colours and, for each $i \in\{1,2\}, x w_{i}$ is not coloured with $\mu, y w_{i}$ is not coloured with $\nu$, and if $x w_{i}$ is coloured with $\nu$ then $y w_{i}$ is not coloured with $\mu$.

Proof. (a) follows from the well-known result [3] that a cycle of even length is 2choosable (or, equivalently, edge-2-choosable). To prove (b), for each $i$ let $L\left(x w_{i}\right):=$ $\Gamma\left(x w_{i}\right) \backslash\{\mu\}$ and $L\left(y w_{i}\right):=\Gamma\left(y w_{i}\right) \backslash\{\nu\}$, so that $|L(z)| \geqslant 2$ for each edge $z$. We may assume that $\nu \in L\left(x w_{i}\right)$ and $\mu \in L\left(y w_{i}\right)$ for at least one $i$, since otherwise we require only 


\begin{tabular}{|c|c|c|c|c|c|c|c|}
\hline Condition & $\begin{array}{l}\text { Colouring } \\
\quad \text { of } u, v\end{array}$ & $\begin{array}{c}\text { For } \\
\text { colc } \\
\text { of }\end{array}$ & $\begin{array}{l}\text { den } \\
\text { ngs } \\
\text { vy }\end{array}$ & Condition & $\begin{array}{l}\text { Colouring } \\
\text { of } u, v\end{array}$ & \multicolumn{2}{|c|}{$\begin{array}{c}\text { Forbidden } \\
\text { colourings } \\
\text { of } u x, v y\end{array}$} \\
\hline A & $\mu \nu$ & $\nu \mu$ & $\xi \xi$ & & & & \\
\hline$B(a, b)$ & $a \nu$ & $\nu a$ & $b a$ & $B^{\prime}(a, b)$ & $\mu b$ & $b \mu$ & $b a$ \\
\hline$C(a, b)$ & $a \nu$ & $\nu b$ & $\xi \xi$ & $C^{\prime}(a, b)$ & $\mu b$ & $a \mu$ & $\xi \xi$ \\
\hline$E(a, b, c)$ & $a \nu$ & $\nu b$ & $c b$ & $E^{\prime}(a, b, c)$ & $\mu b$ & $a \mu$ & $a c$ \\
\hline$G(a, b, c)$ & $a c$ & $c a$ & $c b$ & $G^{\prime}(a, b, c)$ & $c b$ & $a c$ & $b c$ \\
\hline$J(a, b, c, d)$ & $d c$ & $c b$ & $c d$ & $J^{\prime}(a, b, c, d)$ & $c d$ & $a c$ & $d c$ \\
\hline$L(a, b, c, d)$ & $a c$ & $c a$ & $c b$ & $L^{\prime}(a, b, c, d)$ & $c b$ & $a c$ & $b c$ \\
\hline$M(a, b, c, d)$ & $a c$ & $c b$ & $c d$ & $M^{\prime}(a, b, c, d)$ & $c b$ & $a c$ & $d c$ \\
\hline$O(a, b, c)$ & $a a$ & $b c$ & $\xi \xi$ & & & & \\
\hline$S(a, b, c, d)$ & $a d$ & $b a$ & $b c$ & $S^{\prime}(a, b, c, d)$ & $a d$ & $b c$ & $d c$ \\
\hline$U(a, b, c, d)$ & a a & $b c$ & $b d$ & $U^{\prime}(a, b, c, d)$ & a a & $b c$ & $d c$ \\
\hline$Z(a, b, c, d)$ & $a d$ & $b c$ & $\xi \xi$ & & & & \\
\hline $\bar{A}(a, b, c, d)$ & $a d$ & $b a$ & $b c$ & $\bar{A}^{\prime}(a, b, c, d)$ & $a d$ & $b c$ & $d c$ \\
\hline $\bar{D}(a, b, c, d, e)$ & $a d$ & $b c$ & $b e$ & $\bar{D}^{\prime}(a, b, c, d, e)$ & $a d$ & $b c$ & $e c$ \\
\hline
\end{tabular}

Table 2

that $x w_{i}$ and $y w_{i}$ should have different colours for each $i$, and the result follows from part (a). So suppose that $\nu \in L\left(x w_{1}\right)$ and $\mu \in L\left(y w_{1}\right)$. If $\mu \in L\left(y w_{2}\right)$, then colour $x w_{1}$ with $\nu$, $y w_{2}$ with $\mu$, and $x w_{2}$ and $y w_{1}$ with colours different from both $\mu$ and $\nu$, which is possible since $\mu \notin L\left(x w_{2}\right)$ and $\nu \notin L\left(y w_{1}\right)$. If however $\mu \notin L\left(y w_{2}\right)$, then colour $y w_{1}$ with $\mu, x w_{1}$ with a colour different from both $\mu$ and $\nu, x w_{2}$ with a colour different from that of $x w_{1}$, and $y w_{2}$ with a colour different from that of $x w_{2}$, and necessarily different from $\mu$.

If $\Lambda$ is a 4-list-assignment to a nontrivial sepachain $G$, labelled as in Fig. 1(a), and $u, v$ are given colours $\lambda_{u}, \lambda_{v}$ from their lists, then we say that $\left(G, \Lambda, \lambda_{u}, \lambda_{v}\right)$ is standard (or, if the lists and colours are clear from the context, we say just that $G$ is standard) if there is at most one pair of colours $\mu \in \Lambda(u x) \backslash\left\{\lambda_{u}\right\}$ and $\nu \in \Lambda(v y) \backslash\left\{\lambda_{v}\right\}$ such that, if $u x, v y$ are given colours $\mu, \nu$ respectively, then this colouring cannot be extended to a total $\Lambda$-colouring of $G$. For more than half of the possible types for $(G, \Lambda)$, it can be seen from Table 1 that every possible colouring of $u, v$ results in $G$ being standard. The exceptions are listed in Table 2. Note that in most of these cases it is possible to change the colour of $u$ or $v$ in such a way that $G$ becomes standard.

Lemma 5. If $G_{1}$ and $G_{2}$ are nontrivial sepachains that are good for total 4-choosability, then the sepachain $G$ in Fig. 1(d) is very good for total 4-choosability.

Proof. Let $\Lambda$ be a 4 -list-assignment to $G$, and let colours $\lambda_{u}, \lambda_{u x}, \lambda_{v y}, \lambda_{v}$ be assigned to $u, u x, v y, v$. At this point there are at least two colours available for use on each of $x, y$ and at least three for use on each of the edges in the set $E_{0}:=\left\{x x_{1}, x x_{2}, y y_{1}, y y_{2}\right\}$. 
Suppose first that we can assign colours to $x$ and $y$ in such a way that both $G_{1}$ and $G_{2}$ are standard. In this case we borrow a trick from [7], which we call the standard trick; note that it can only be used after $x$ and $y$ have both been coloured. For each $z \in E_{0}$, let $L(z)$ denote the set of colours that can now be used on $z$, where $|L(z)| \geqslant 2$. For each $i$, if there is an ordered pair $\mu_{i} \in L\left(x x_{i}\right), \nu_{i} \in L\left(y y_{i}\right)$ of colours that is forbidden for $x x_{i}, y y_{i}$, and $\mu_{i} \neq \nu_{i}$, then choose a new colour $\xi_{i}$, not contained in any other list, and set $L^{\prime}\left(x x_{i}\right):=L\left(x x_{i}\right) \cup\left\{\xi_{i}\right\} \backslash\left\{\mu_{i}\right\}$ and $L^{\prime}\left(y y_{i}\right):=L\left(y y_{i}\right) \cup\left\{\xi_{i}\right\} \backslash\left\{\nu_{i}\right\}$ (we call this the standard construction); otherwise, set $L^{\prime}\left(x x_{i}\right):=L\left(x x_{i}\right)$ and $L^{\prime}\left(y y_{i}\right):=L\left(y y_{i}\right)$. Identify $x_{i}, y_{i}$ into a new vertex $w_{i}$. By Lemma 4(a), the edges of the 4-cycle $x w_{1} y w_{2} x$ can be coloured from the lists $L^{\prime}$. Transfer this colouring to $G$ (giving edges $x x_{i}, y y_{i}$ the colours of $\left.x w_{i}, y w_{i}\right)$. For each $i$, at most one of the edges $x x_{i}, y y_{i}$ is coloured with the new colour $\xi_{i}$. If, say, $x x_{i}$ is, then uncolour $x x_{i}$ and recolour it with a colour that is not used on $u x$, $x$ or $x x_{3-i}$; perhaps it will now have the same colour as $y y_{i}$, but that is acceptable. In this way we can colour the edges in $E_{0}$ so that neither pair $x x_{i}, y y_{i}$ is given its forbidden pair of colours (if it has one), and so this colouring can be extended to both graphs $G_{i}$ so as to form a total $\Lambda$-colouring of $G$.

It is clear from Table 2 that this proves the result unless one of $G_{1}$ and $G_{2}$ has type $A, B, C, E, B^{\prime}, C^{\prime}$ or $E^{\prime}$, since in all other cases we can choose a colour for $x\left(=u_{1}\right)$ such that $G_{1}$ is standard whatever the colour of $y\left(=v_{1}\right)$, and we can choose a colour for $y$ $\left(=v_{2}\right)$ such that $G_{2}$ is standard whatever the colour of $x\left(=u_{2}\right)$. It also proves the result if $G_{1}$, say, has type $B, C$ or $E$ (or $B^{\prime}, C^{\prime}$ or $E^{\prime}$ ) and $G_{2}$ has any type other than $A, B, C$ or $E$ (or $A, B^{\prime}, C^{\prime}$ or $E^{\prime}$ ), for the same reason. There remain two cases to consider.

Case 1: $G_{1}$ (say) has type $A$.

Suppose first that $G_{2}$ also has type $A$. Colour $x, y$ with colours $\mu, \nu$ from their lists, and identify $x_{i}, y_{i}$ into a new vertex $w_{i}$, for each $i$. Colour the edges of the 4-cycle $x w_{1} y w_{2} x$ as in Lemma $4(\mathrm{~b})$, and transfer this colouring to $G$ (giving edges $x x_{i}, y y_{i}$ the colours of $\left.x w_{i}, y w_{i}\right)$. Then all the requirements of Condition $A$ are satisfied for both $G_{1}$ and $G_{2}$, and so this colouring can be extended to a total $\Lambda$-colouring of $G$.

If $G_{2}$ has any type other than $A$, then it is possible to assign colours to $x, y$ in such a way that $G_{2}$ is standard. Using the standard construction, we can modify the lists so that the colouring can be extended to $G_{2}$ as long as $x x_{2}$ and $y y_{2}$ do not have the same colour. The result now again follows from Lemma 4(b), used as in the previous paragraph.

Case 2a: $G_{1}$ has type $B(a, b), C(a, b)$ or $E(a, b, c)$ and $G_{2}$ has type $B\left(a^{\prime}, b^{\prime}\right), C\left(a^{\prime}, b^{\prime}\right)$ or $E\left(a^{\prime}, b^{\prime}, c^{\prime}\right)$.

Case 2b: $G_{1}$ has type $B^{\prime}(a, b), C^{\prime}(a, b)$ or $E^{\prime}(a, b, c)$ and $G_{2}$ has type $B^{\prime}\left(a^{\prime}, b^{\prime}\right), C^{\prime}\left(a^{\prime}, b^{\prime}\right)$ or $E^{\prime}\left(a^{\prime}, b^{\prime}, c^{\prime}\right)$.

Case $2 \mathrm{~b}$ is the same as Case 2a reflected left-to-right, and so we will consider only Case 2a. Give $x, y$ colours from their lists so that $x\left(=u_{2}\right)$ is not given colour $a^{\prime}$. Then $G_{2}$ is standard; carry out the standard construction on $G_{2}$ if necessary, so that it suffices for $x x_{2}$ and $y y_{2}$ to be given different colours. We may assume that the colour of $x$ is $a$ (so that $\left.a \neq a^{\prime}\right)$, since otherwise $G_{1}$ is standard as well. There are now at least two colours available for each of the edges in $E_{0}$. 
If $G_{1}$ has type $B(a, b)$, then give $y y_{1}\left(=v_{1} y_{1}\right)$ a colour different from $a$, then colour $y y_{2}$ (differently from $y y_{1}$ ), $x x_{2}$ (differently from $y y_{2}$ ) and $x x_{1}$ (differently from $x x_{2}$; possibly $x x_{1}$ has the same colour as $y y_{1}$, but this does not matter). This colouring can be extended to a total $\Lambda$-colouring of $G$. If $G_{1}$ has type $E(a, b, c)$ then the same argument works if we start by giving $y y_{1}$ a colour different from $b$. Thus we may assume that $G_{1}$ has type $C(a, b)$. By interchanging $G_{1}$ and $G_{2}$ if necessary, we may assume that $G_{2}$ also has type $C$, specifically $C\left(a^{\prime}, b^{\prime}\right)$. However, since $x\left(=u_{2}\right)$ does not have colour $a^{\prime}$, it can be seen from Table 1 that condition $C\left(a^{\prime}, b^{\prime}\right)$ imposes no restriction on the colours of $x x_{2}\left(=u_{2} x_{2}\right)$ and $y y_{2}\left(=v_{2} y_{2}\right)$. So colour $x x_{1}$ differently from $y$, and $y y_{1}$ differently from $x x_{1}$, then $x x_{2}$ differently from $x x_{1}$, and $y y_{2}$ differently from $y y_{1}$. This colouring can be extended to a total $\Lambda$-colouring of $G$, and this completes the proof of Lemma 5 .

Lemma 6. If a nontrivial sepachain $G_{1}$ is good for total 4-choosability, then the sepachain $G$ in Fig. 1(f) is very good for total 4-choosability.

Proof. Let $\Lambda$ be a 4-list-assignment to $G$, and let colours $\lambda_{u}, \lambda_{u x}, \lambda_{v y}, \lambda_{v}$ be assigned to $u, u x, v y, v$. For each uncoloured element $z$, let $L(z)$ denote the set of colours that can now be used on $z$, so that $|L(z)| \geqslant 2$ if $z \in\{x, y\},|L(z)| \geqslant 3$ if $z \in E_{0}:=\left\{x s, y s, x x_{1}, y y_{1}\right\}$, and $|L(s)|=4$. There are two cases to consider, which are dealt with by rather similar arguments.

Case 1: $G_{1}$ has type $A$.

If we colour $x$ and $y$ with arbitrary colours $\mu, \nu$ from their lists, and identify $x_{1}, y_{1}$ into a new vertex $w_{1}$, then it follows from Lemma 4 (b) that we can colour the four edges in a way that meets all the required conditions. The only problem is if $x, x s, y s, y$ have been given the four distinct colours in $L(s)$. This will not happen if $x$ and $y$ are given the same colour, or if one of them has been given a colour not in $L(s)$, and so we may assume that this is not possible; specifically, we assume that

$$
L(x)=\{a, b\}, \quad L(y)=\{c, d\}, \quad L(s)=\{a, b, c, d\} .
$$

If it is possible to colour $x, x s, y$ so that $x s$ and $y$ have the same colour, do so, w.l.o.g. with colours $a, c, c$, then colour $x x_{1}, y y_{1}, y s, s$ in that order, which is possible since both $y s$ and $s$ have two neighbours with the same colour. (Specifically, we colour $x x_{1}$ with a colour $c_{1} \in L\left(x x_{1}\right) \backslash\{a, c\}, y y_{1}$ with $c_{2} \in L\left(y y_{1}\right) \backslash\left\{c, c_{1}\right\}$, ys with $c_{3} \in L(y s) \backslash\left\{c, c_{2}\right\}$, and $s$ with $c_{4} \in L(s) \backslash\left\{a, c, c_{3}\right\}$.) In view of this, we may suppose that $L(x s) \cap L(y)=\emptyset$, and similarly that $L(x) \cap L(y s)=\emptyset$. Thus there exist colours $e \in L(x s) \backslash L(s)$ and $e^{\prime} \in L(y s) \backslash L(s)$, where possibly $e=e^{\prime}$.

Now colour $x$ and $y$ with arbitrary colours $\mu, \nu$ from their lists, and extend this colouring to the edges in $E_{0}$ using Lemma 4(b). If $x s$ or $y s$ is coloured with $e$ or $e^{\prime}$ or any other colour not in $L(s)$, then this colouring can be extended to $s$ and hence to a total $\Lambda$-colouring of $G$. So we may assume that this is not so, and that

$$
L(x s)=\{a, b, e\}, \quad L(y s)=\left\{c, d, e^{\prime}\right\}, \quad L(x s) \cap L(y s)=\emptyset,
$$


and $x x_{1}, y y_{1}$ are coloured with $e, e^{\prime}$ respectively (so that $e \neq e^{\prime}$ ), since otherwise we could recolour $x s$ or $y s$ with $e$ or $e^{\prime}$ or some other colour not in $L(s)$, without changing the colour of any other element. But now we can recolour $y y_{1}$ with a colour $c_{1} \in L\left(y y_{1}\right) \backslash\left\{e, e^{\prime}\right\}, y$ with a colour in $\{c, d\} \backslash\left\{c_{1}\right\}$, and $y s$ with $e^{\prime}$, and this colouring can be extended to $s$ and then to a total $\Lambda$-colouring of $G$.

Case 2: $G_{1}$ does not have type $A$.

By reflecting left-right if necessary, we may assume that $G_{1}$ does not have any of the types in the right half of Table 2 , so that it is possible to colour $x\left(=u_{1}\right)$ in such a way that, whatever colour is given to $y\left(=v_{1}\right), G_{1}$ is standard. If this is done, then the standard trick together with Lemma 4(a) shows that the edges in $E_{0}$ can be coloured so that all conditions are satisfied. As in Case 1, the only problem is if $x, x s, y s, y$ have been given the four distinct colours in $L(s)$. This will not happen if $x$ and $y$ are given the same colour, or if one of them has been given a colour not in $L(s)$, and so we may assume that this is not possible; specifically, we assume that

$$
x \text { has colour } a, \quad L(y)=\{c, d\}, \quad L(s)=\{a, b, c, d\} .
$$

If it is possible to colour $x s$ and $y$ with the same colour (and $x$ with $a$ ), do so, then colour $x x_{1}, y y_{1}, y s, s$ in that order, which is possible since both $y s$ and $s$ have two neighbours with the same colour. (The colour $c_{1}$ given to $x x_{1}$ rules out at most one colour $c_{2}$ for $y y_{1}$, if $\left(c_{1}, c_{2}\right)$ is the forbidden ordered pair; it is irrelevant whether or not $c_{1}=c_{2}$.) In view of this, we may suppose that $L(x s) \cap L(y)=\emptyset$, so that there is at least one colour $e \in L(x s) \backslash L(s)$.

Now colour $x$ with $a$ and $y$ with an arbitrary colour $\nu \in L(y)$, and extend this colouring to the edges in $E_{0}$ using the standard trick and Lemma 4(a). If $x s$ or ys is coloured with $e$ or any other colour not in $L(s)$, then this colouring can be extended to $s$ and hence to a total $\Lambda$-colouring of $G$. So we may assume that this is not so.

Suppose first that $e \in L(y s)$. Then we can change the colour of $x s$ or ys to $e$ without changing any other colour, unless $x x_{1}$ and $y y_{1}$ both have colour $e$. (This is after completing the standard trick, when $x x_{1}$ and $y y_{1}$ may have the same colour.) In that case, recolour $x s$ with $e$ and $x x_{1}$ with a colour in $L\left(x x_{1}\right) \backslash\{a, e\}$; if this would cause $x x_{1}$ and $y y_{1}$ to have the forbidden pair of colours, then, instead, recolour $y s$ with $e$ and $y y_{1}$ with a colour in $L\left(y y_{1}\right) \backslash\{\nu, e\}$, and note that now $x x_{1}$ and $y y_{1}$ cannot have the forbidden ordered pair of colours. So the colouring can be extended to $s$ and then to a total $\Lambda$-colouring of $G$.

So we may assume that $e \notin L(y s)$. Now recolour $x s$ with $e, x x_{1}$ with a colour $c_{1} \in L\left(x x_{1}\right) \backslash\{a, e\}, y y_{1}$ with a colour $c_{2} \in L\left(y y_{1}\right) \backslash\{\nu\}$ such that $\left(c_{1}, c_{2}\right)$ is not the forbidden ordered pair of colours, and $y s$ with a colour in $L(y s) \backslash\left\{\nu, c_{2}\right\}$. This colouring can be extended to $s$ and then to a total $\Lambda$-colouring of $G$, and this completes the proof of Lemma 6.

The following lemma is a special case of a result in [11], where it is proved by an entirely different method. For completeness, we include here a proof using the methods of the current paper.

Lemma 7. The sepachain G in Fig. 1(e) is very good for total 4-choosability. 
Proof. Let $\Lambda$ be a 4-list-assignment to $G$, and let colours $\lambda_{u}, \lambda_{u x}, \lambda_{v y}, \lambda_{v}$ be assigned to $u, u x, v y, v$. For each uncoloured element $z$, let $L(z)$ denote a set of colours that can now be used on $z$, where

$$
|L(z)|= \begin{cases}2 & \text { if } z=x \text { or } y \\ 3 & \text { if } z \text { is an edge } \\ 4 & \text { if } z=r \text { or } s\end{cases}
$$

Note that if we first colour $x$ and $y$, then we can colour the four edges by Lemma 4(a) since each edge has at least two usable colours in its list, and we can then colour $r$ and $s$ unless either $x, x r, r y, y$ have been coloured with the four distinct colours in $L(r)$, or else $x, x s, s y, y$ have been coloured with the four distinct colours in $L(s)$. This will not happen if we can give $x$ and $y$ the same colour, and so we may assume that $L(x) \cap L(y)=\emptyset$; specifically, we choose

$$
L(x)=\{a, b\} \quad \text { and } \quad L(y)=\{c, d\} .
$$

The same method of colouring will work if we can give $x$ or $y$ a colour that is not in $L(r) \cup L(s)$, and so we may assume that

$$
\{a, b, c, d\} \subseteq L(r) \cup L(s)
$$

It will also work if we can give $x$ a colour not in $L(r)$ and $y$ a colour not in $L(s)$, or vice versa, and so we may assume that

$$
L(x) \subset L(r) \text { or } L(y) \subset L(s), \quad \text { and } \quad L(x) \subset L(s) \text { or } L(y) \subset L(r) .
$$

Suppose that $L(x) \cap L(r y) \neq \emptyset$. Then colour $x$ and $r y$ with the same colour $\mu$, and let $L^{\prime}(z):=L(z) \backslash\{\mu\}$ for each uncoloured element $z$. By (1) and (2), $\left|L^{\prime}(z)\right| \geqslant 2$ for each $z$, and $\left|L^{\prime}(z)\right| \geqslant 3$ if $z \in\{r, s\}$. If it is now possible to colour $x s$ and $y$ with the same colour, do so, and then colour $x r, r, s y, s$ in that order. If however this is not possible, then either $\left|L^{\prime}(s)\right|=4$, or else $L^{\prime}(x s)$ or $L^{\prime}(y)$ contains a colour not in $L^{\prime}(s)$; in either case, colour $x s, s y$ and $y$ in that order, using a colour not in $L^{\prime}(s)$ if possible, and then colour $s, x r$ and $r$ in that order. In view of this and symmetric arguments, we may assume that

$$
L(x) \cap L(r y)=L(x) \cap L(s y)=L(y) \cap L(x r)=L(y) \cap L(x s)=\emptyset .
$$

If $L(r)=L(s)=\{a, b, c, d\}$, then by (1), (2) and (5) we can colour $x r$ with a colour $f \notin$ $L(r)$ and $s y$ with a colour $f^{\prime} \notin L(s)$, and we can then colour the elements $x s, r y, x, y, r, s$ in that order, since $f \notin L(x)$ and $f^{\prime} \notin L(y)$ by $(2)$.

If $L(r)=\{a, b, c, d\}$ and $L(s) \neq\{a, b, c, d\}$, say $d \notin L(s)$, then we can colour $x r$ with a colour $f \notin L(r)$ and $y$ with the colour $d \notin L(s)$, and we can then colour the elements $r y, s y, x s, x, r, s$ in that order, since $f \notin L(x)$.

So we may assume that $L(r) \neq\{a, b, c, d\}$ and $L(s) \neq\{a, b, c, d\}$. By (1)-(4) and symmetry we may therefore assume that $L(r)=\{a, b, c, e\}$ and $L(s)=\left\{a, b, d, e^{\prime}\right\}$, where possibly $e=e^{\prime}$ but $e, e^{\prime} \notin\{a, b, c, d\}$.

Note that if three mutually adjacent elements each have a list of two colours, then they can be coloured from these lists unless the lists are all identical. Thus if we colour 
$r y$ and $s y$ with colours $p$ and $q$, then we can extend this colouring to $x r, x$ and $x s$ unless $L(x r)=\{a, b, p\}$ and $L(x s)=\{a, b, q\}$. We will use this idea in conjunction with the following table, which we will explain shortly.

Colouring for

$r y, y, s y$

$\begin{array}{lll}f d c & \text { or } & d c g \\ f d c & \text { or } & f c d \\ f d c & \text { or } & f c g \\ f c d & \text { or } & f c g \\ f c g_{1} & \text { or } & f c g_{2}\end{array}$

At least one

extends because

$d \notin L(r)$ and $c \notin L(s)$.

$f \notin L(r)$ and $c \notin L(s)$.

$f \notin L(r)$ and $c \notin L(s)$.

$f \notin L(r)$ and $c \notin L(s)$.

$f \notin L(r)$ and $c \notin L(s)$.

By (2) and (5), $\{a, b\} \cap L(r y)=\emptyset$, and so there is a colour $f \in L(r y) \backslash\{a, b, c, d\}$. Similarly, there is a colour $g \in L(s y) \backslash\{a, b, c, d\}$, where possibly $g=f$. If $d \in L(r y)$ and $c \in L(s y)$, then we can colour $r y, y, s y$ respectively with either $f, d, c$ or $d, c, g$ as in row (i) of the table; at least one of these colourings can be extended to $x r, x, x s$ as explained in the previous paragraph (since $L(x s)$ cannot equal both $\{a, b, c\}$ and $\{a, b, g\}$ ), and then to $r$ and $s$ since $d \notin L(r)$ and $c \notin L(s)$. So we may suppose that $d \notin L(r y)$ or $c \notin L(s y)$, w.l.o.g. $d \notin L(r y)$. It follows that there are at least two possible choices for $f \in L(r y) \backslash\{a, b, c, d\}$, and we can ensure $f \neq e$, so that $f \notin L(r)$. If $\{c, d\} \subset L(s y)$, we can complete the colouring by using one of the schemes for $r y, y, s y$ in row (ii) of the table; thus we may assume that $\{c, d\} \not \subset L(s y)$, which means that there are at least two choices for $g$, and we can choose $g \neq f$. Now if $c \in L(s y)$ or $d \in L(s y)$ we can complete the colouring using row (iii) or (iv) of the table respectively, and so we may assume that neither of these happens. This means that there are three choices for $g$, and we can choose distinct colours $g_{1}, g_{2} \in L(s y) \backslash\{a, b, c, d, f\}$. Finally we can complete the colouring using row (v) of the table, and this completes the proof of Lemma 7.

\section{The harder parallel constructions}

Lemma 8. The sepachain G in Fig. 1(g) is good for total 4-choosability.

Proof. Let $\Lambda$ be a 4-list-assignment to $G$, and let colours $\lambda_{u}, \lambda_{u x}, \lambda_{v y}, \lambda_{v}$ be assigned to $u, u x, v y, v$. For each uncoloured element $z$, let $\widehat{L}(z)$ denote the set of all colours that can now be used on $z$, and let $L(z) \subseteq \widehat{L}(z)$ where

$$
|L(z)|= \begin{cases}2 & \text { if } z=x, x y \text { or } y \\ 3 & \text { if } z=w x \text { or } w y \\ 4 & \text { if } z=w\end{cases}
$$

Up to left-right reflections, the lists $L(x), L(x y), L(y)$ fall into one of the patterns (i)-(xii) shown in Table 3 (where for brevity we have written $a b$ instead of $\{a, b\}$, etc.). Here (i) is the pattern with all three lists the same, (ii)-(v) are the patterns with two lists the same 


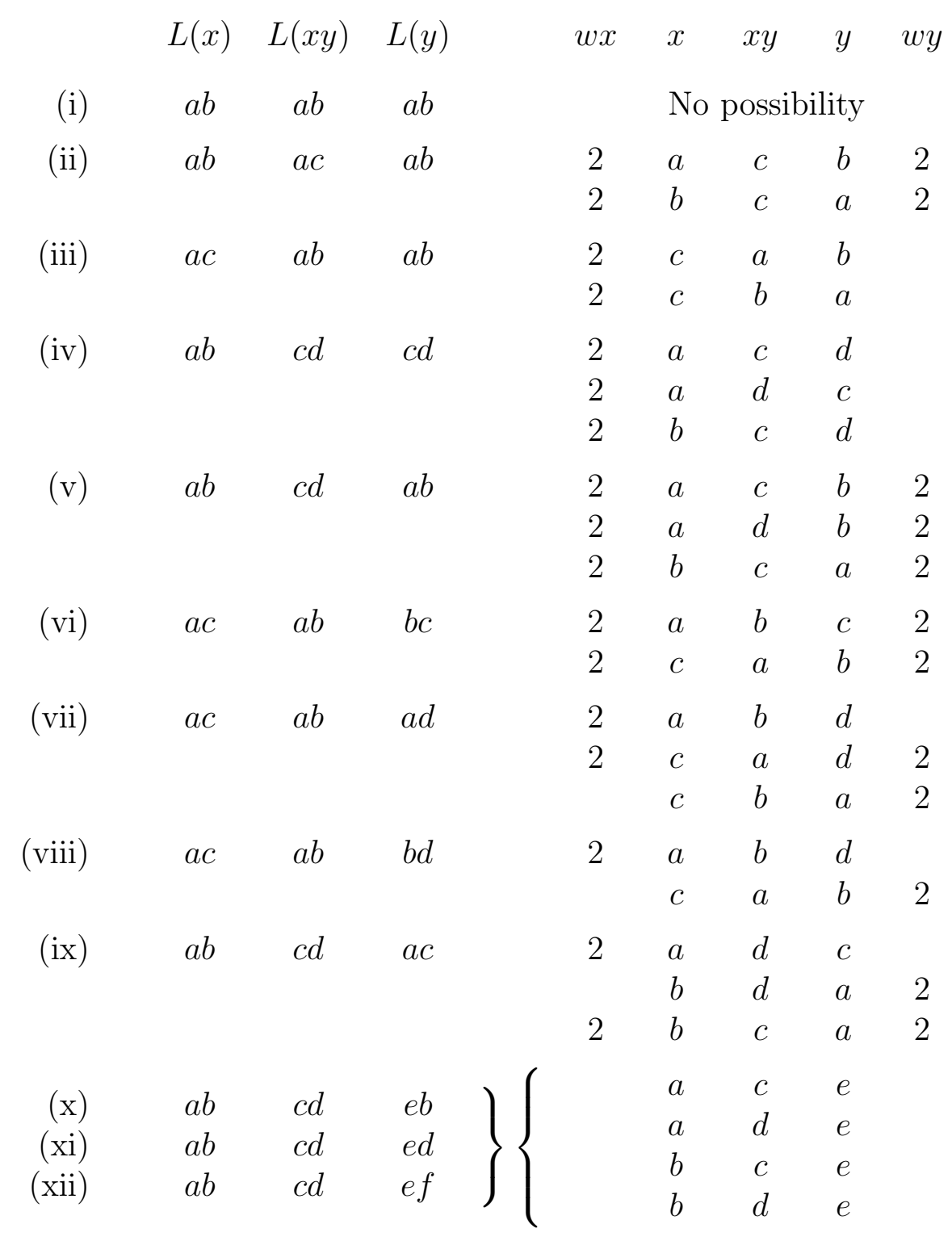

Table 3 


$\begin{array}{cccccc}\text { Row } & \Lambda(x) & \Lambda(x y) & \Lambda(y) & \begin{array}{c}\text { Bad colourings } \\ \lambda_{u} \lambda_{u x} \lambda_{v y} \lambda_{v}\end{array} & \begin{array}{c}\text { Avoided by } \\ \text { condition }\end{array} \\ 1 & \{a, b, c, d\} & \{a, b, c, d\} & \{a, b, c, d\} & \mu \nu \mu \nu & A \\ 2 & \{a, b, c, d\} & \{a, b, c, d\} & \{a, b, d, e\} & \mu c \mu e & C^{\prime}(c, e) \\ 3 & \{a, b, c, e\} & \{a, b, c, d\} & \{a, b, c, d\} & e \nu d \nu & C(e, d) \\ 4 & \{a, b, c, e\} & \{a, b, c, d\} & \{a, b, d, e\} & e c d e & D(c, d) \\ 5 & \{a, b, c, e\} & \{a, b, c, d\} & \{a, b, d, f\} & e c d f & D(c, d)\end{array}$

Table 4

and the third different, and (vi)-(xii) are those with no two lists the same; of these, (vi) is the only one where the union of the lists contains exactly three colours, (vii)-(ix) are where it contains four colours, (x) and (xi) contain five colours, and (xii) has six colours.

For each pattern, some of the ways of colouring $x, x y, y$ from their lists are also shown in Table 3. After such a colouring, there is at least one colour available for $w x$, at least one for $w y$, and at least two for $w$. If $w y$ can be given the same colour as $x$, then the colouring can be extended to $w x$ and then $w$ to give the required total colouring of $G$. We will assume henceforth that this is not possible. This means that if there is a colouring in which $x$ is coloured with $a$, say, then we may assume that $a \notin L(w y)$, and so if there is a different colouring in which $x y$ or $y$ is coloured with $a$, then this second colouring can be extended to $w y$ in at least two different ways; this is indicated by a figure 2 in the column for $w y$, and it means that if we colour $w x$ first, we can then colour $w y$ differently from $w x$. The same holds with $x$ and $y$ interchanged, as indicated by a figure 2 in the column for $w x$. It follows that all the colourings listed for the patterns (ii)-(ix) can be extended to $w x$ and $w y$.

Now suppose that for one of these patterns there is a colouring shown in which $x y$ is coloured with $a$, say, and a second colouring in which $x$ or $y$ is coloured with $a$. If $a \in L(w)$ then the first colouring can be extended to $w$ by giving $w$ colour $a$, while if $a \notin L(w)$ then the second colouring can be extended because one of the four neighbours of $w$ has a colour not in $L(w)$. This argument shows that the patterns in (iii), (iv) and (vi)-(ix) all allow for total colourings of $G$, whatever lists have been assigned to $w x, w y$ and $w$. For pattern (v), we may assume by the same argument that $c, d \notin L(w)$, so that if $w y$ can be coloured with $c$ or $d$ then $w x$ and $w$ can be coloured. But if $L(w y)$ does not contain $c$ or $d$, then, since we are already assuming it does not contain $a$ or $b$, we can colour $w x$ and $w$ first and still have a colour to give to $w y$. Thus we have shown that all the patterns in (iii)-(ix) allow for total colourings of $G$.

We can deal with patterns (x)-(xii) all together. Suppose first that $a \notin L(w x)$. Then the colourings $a, c, e$ and $a, d, e$ of $x, x y, y$ both extend to $w x$ after $w y$. By a previous argument we may therefore suppose that $c, d \notin L(w)$. If $c \in L(w x)$ then we can use $c$ on $w x$ or $w y$ in the second of these extensions and then colour $w$; and if $c \notin L(w x)$ then, since $a \notin L(w x)$, we can use the colouring $a, c, e$ and then colour $w y, w, w x$ in that 


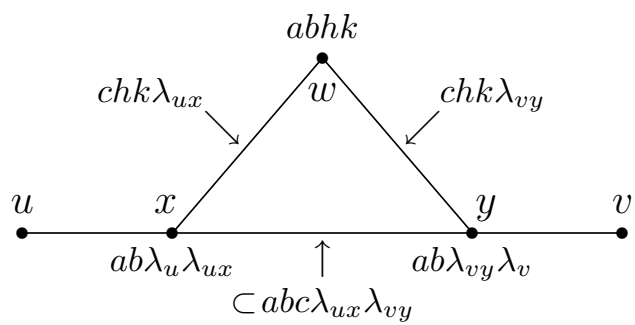

(a)

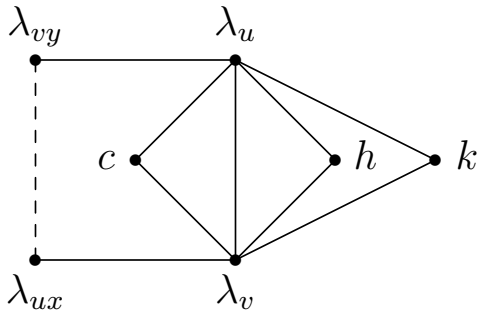

(b)

Fig. 3

order. So we may assume that $a \in L(w x)$, and similarly $b \in L(w x)$. Therefore one of $c, d$, w.l.o.g. $c, \notin L(w x)$. Thus the colourings $a, c, e$ and $b, c, e$ of $x, x y, y$ both extend to $w x$ after $w y$, and one of them can be extended to $w$ unless $L(w)=\left\{a, b, e, d^{\prime}\right\}$ for some colour $d^{\prime} \notin\{a, b, c, e\}$, and $L(w x)=\left\{a, b, d^{\prime}\right\}$ and $L(w y)=\left\{c, e, d^{\prime}\right\}$. But then we can colour $x, x y, y$ with $a, d, e$ and $w x, w, w y$ with $b, d^{\prime}, c$ (whether or not $d^{\prime}=d$ ). This proves the result for patterns (x)-(xii).

We have now dealt with patterns (iii)-(xii), and so we must consider patterns (i) and (ii). We deal with (i) first. When we chose $L(x), L(x y), L(y)$ at the start of the proof, it is not possible that we were forced into pattern (i) unless $\widehat{L}(x)=\widehat{L}(x y)=\widehat{L}(y)=\{a, b\}$, which implies $\lambda_{u x} \neq \lambda_{v y}$, say $\lambda_{u x}=c$ and $\lambda_{v y}=d$, and $\Lambda(x)=\left\{a, b, c, \lambda_{u}\right\}, \Lambda(x y)=$ $\{a, b, c, d\}$, and $\Lambda(y)=\left\{a, b, d, \lambda_{v}\right\}$. The essentially different possibilities for $\left(\lambda_{u}, \lambda_{v}\right)$ are then $(d, c),(d, e),(e, c),(e, e)$ and $(e, f)$ (where distinct letters represent distinct colours), giving the lists shown in Table 4 . In each case, if a (possibly different) assignment of colours $\lambda_{u}, \lambda_{u x}, \lambda_{v y}, \lambda_{v}$ to $u, u x, v y, v$ puts us into pattern (i), that is,

$$
\Lambda(x) \backslash\left\{\lambda_{u}, \lambda_{u x}\right\}=\Lambda(x y) \backslash\left\{\lambda_{u x}, \lambda_{v y}\right\}=\Lambda(y) \backslash\left\{\lambda_{v y}, \lambda_{v}\right\}=X, \text { say }
$$

where $|X|=2$, then this 'bad colouring' $\lambda_{u}, \lambda_{u x}, \lambda_{v y}, \lambda_{v}$ must match the pattern shown in the penultimate column of Table 4 , and it can be avoided by imposing the condition given in the final column.

We now deal with pattern (ii). When we chose $L(x), L(x y), L(y)$ at the start of the proof, if we were forced into pattern (ii), or the only way of avoiding it would put us into pattern (i) instead, then $\widehat{L}(x)=\widehat{L}(y)=\{a, b\}$ and $c \in \widehat{L}(x y) \subseteq\{a, b, c\}$. By arguments similar to those used above, one of the two colourings in (ii) will extend to the whole of $G$ if $c \in \widehat{L}(w)$ or if $a$ or $b \in \widehat{L}(w x)$ or $\widehat{L}(w y)$. It is not difficult to see that neither colouring extends if and only if the lists are as shown in Fig. 3(a). Here the colours $a, b, c$ are distinct by definition, but the only restriction on the other letters is that each list must contain four distinct colours. The pairs of letters that could represent the same colour are shown by the edges in the graph in Fig. 3(b). So $h, \lambda_{u}, \lambda_{v}$ could all be equal, for example; or $\lambda_{u}$ could equal $c$ or $\lambda_{v y}$ and/or $\lambda_{v}$ could equal $c$ or $\lambda_{u x}$; and $\lambda_{u x}$ can equal $\lambda_{v y}$ only if $\{a, b, c\} \subset \Lambda(x y)$. Various pairs of colours are determined by the lists, as follows: 


\begin{tabular}{|c|c|c|c|c|c|c|c|}
\hline$\left(\lambda_{u}, \lambda_{v}\right)$ & $\Lambda(x)$ & $\Lambda(x y)$ & $\Lambda(y)$ & $\begin{array}{l}\text { Colourings } \\
\lambda_{u} \lambda_{u x} \lambda_{v y} \lambda_{v} \\
\text { bad for (ii) }\end{array}$ & $\begin{array}{l}\text { Row of } \\
\text { Table } 4\end{array}$ & $\begin{array}{l}\text { Colourings } \\
\lambda_{u} \lambda_{u x} \lambda_{v y} \lambda_{v} \\
\text { bad for (i) }\end{array}$ & $\begin{array}{c}\text { Avoided by } \\
\text { condition }\end{array}$ \\
\hline$(c, c)$ & $\begin{array}{l}a b d c \\
a b d c \\
a b d c\end{array}$ & $\begin{array}{l}a b c d \\
a b c e \\
a c d e\end{array}$ & $\begin{array}{l}a b e c \\
a b e c \\
a b e c\end{array}$ & $\begin{array}{l}\text { cdec } \\
\text { cdec } \\
\text { cdec }\end{array}$ & $\begin{array}{l}2 \\
3 \\
4\end{array}$ & $\begin{array}{l}\mu d \mu e \\
d \nu e \nu \\
b d e b\end{array}$ & $\begin{array}{l}D^{\prime}(d, e) \\
D(d, e) \\
D(d, e)\end{array}$ \\
\hline$(c, d)$ & $\begin{array}{l}a b d c \\
a b d c \\
a b d c\end{array}$ & $\begin{array}{l}a b c d \\
a b c e \\
a c d e\end{array}$ & $\begin{array}{l}\text { abed } \\
\text { abed } \\
\text { abed }\end{array}$ & $\begin{array}{l}\text { cded } \\
\text { cded } \\
\text { cded }\end{array}$ & $\begin{array}{l}2 \\
4 \\
4\end{array}$ & $\begin{array}{l}\mu c \mu e \\
d c e d \\
b c e b\end{array}$ & $\begin{array}{c}F^{\prime}(c, e, d) \\
P^{\prime}(d, c, e) \\
D(c, e)\end{array}$ \\
\hline$(c, f)$ & $\begin{array}{l}a b d c \\
a b d c \\
a b d c\end{array}$ & $\begin{array}{l}a b c d \\
a b c e \\
a c d e\end{array}$ & $\begin{array}{l}\text { abef } \\
\text { abef } \\
\text { abef }\end{array}$ & $\begin{array}{l}c d e f \\
c d e f \\
c d e f\end{array}$ & $\begin{array}{l}- \\
5 \\
-\end{array}$ & $d c e f$ & $\bar{B}^{\prime}(c, d, e, f)$ \\
\hline$(e, d)$ & $\begin{array}{l}a b d e \\
\text { abde } \\
\text { abde }\end{array}$ & $\begin{array}{l}a b c d \\
a b c e \\
a c d e\end{array}$ & $\begin{array}{l}\text { abed } \\
\text { abed } \\
\text { abed }\end{array}$ & $\begin{array}{l}\text { eded } \\
\text { eded } \\
\text { eded }\end{array}$ & $\begin{array}{l}- \\
- \\
-\end{array}$ & & \\
\hline$(e, f)$ & $\begin{array}{l}\text { abde } \\
\text { abde } \\
\text { abde }\end{array}$ & $\begin{array}{l}a b c d \\
a b c e \\
a c d e\end{array}$ & $\begin{array}{l}\text { abef } \\
\text { abef } \\
\text { abef }\end{array}$ & $\begin{array}{l}\text { edef } \\
\text { edef } \\
\text { edef }\end{array}$ & $\begin{array}{l}- \\
- \\
-\end{array}$ & & \\
\hline Case 2 & $a b d e$ & $a b c d$ & $a b c d$ & $e d d c$ & 3 & $e \nu c \nu$ & $C(e, c)$ \\
\hline Case 3 & $a b c d$ & $a b c d$ & $a b c d$ & $\left.\begin{array}{l}c d d c \\
d c c d\end{array}\right\}$ & 1 & $\mu \nu \mu \nu$ & $A$ \\
\hline
\end{tabular}

Table 5

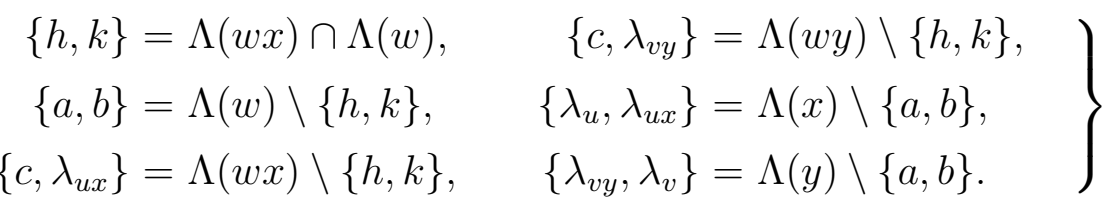

We now distinguish three cases.

Case 1: $\Lambda(w x) \neq \Lambda(w y)$.

Then $\lambda_{u x} \neq \lambda_{v y}$ and $\{c\}=(\Lambda(w x) \cap \Lambda(w y)) \backslash\{h, k\}$, so that $\lambda_{u}, \lambda_{u x}, \lambda_{v y}, \lambda_{v}$ are uniquely determined by (6). This shows that there is only one colouring of $u, u x, v y, v$ that will not extend to the whole of $G$ because it gives rise to pattern (ii), although there may be other colourings that fail to extend because they give rise to pattern (i). We can avoid the colouring that is bad for pattern (ii) by imposing an appropriate condition on the colours of $u, u x, v y, v$ (condition $D\left(\lambda_{u x}, \lambda_{v y}\right)$, for example), but we need to show that this condition can be chosen so as to avoid also any colourings that give rise to pattern (i). 
For convenience write $\lambda_{u x}$ as $d$ and $\lambda_{v y}$ as $e$, so that $\Lambda(x)=\left\{a, b, d, \lambda_{u}\right\}$ and $\Lambda(y)=$ $\left\{a, b, e, \lambda_{v}\right\}$. Since $c \in \Lambda(x y) \subset\{a, b, c, d, e\}$, we may assume that $\Lambda(x y)=\{a, b, c, d\}$ or $\{a, b, c, e\}$ or $\{a, c, d, e\}$. (Note that $\{b, c, d, e\}$ would be essentially the same as $\{a, c, d, e\}$, since $a$ and $b$ are interchangeable.) In each row of Table $4,|\Lambda(x) \cup \Lambda(x y) \cup \Lambda(y)| \leqslant 6$, with equality only in row 5 , where $|\Lambda(x) \cap \Lambda(y)|=2$. It therefore suffices to consider the following five essentially different possibilities for $\left(\lambda_{u}, \lambda_{v}\right):(c, c),(c, d),(c, f),(e, d)$ and $(e, f)$ (where distinct letters represent distinct colours; note that $(e, c),(f, c)$ and $(f, d)$ would be essentially the same as $(c, d),(c, f)$ and $(e, f)$, respectively, under leftright reflection). The corresponding sets $\Lambda(x), \Lambda(x y), \Lambda(y)$ are listed in the top 15 lines of Table 5 (omitting braces and commas for brevity), together with the colourings of $u, u x, v y, v$ that are bad for pattern (ii) or for pattern (i). Note that in the first row of Table 5, for example, choosing $\left(\lambda_{u}, \lambda_{u x}, \lambda_{v y}, \lambda_{v}\right)=(a, d, e, a)$ or $(b, d, e, b)$ instead of $(c, d, e, c)$ would put us into pattern (ii) with $\widehat{L}(x)=\widehat{L}(y)=\{b, c\}$ or $\{a, c\}$ in place of $\{a, b\}$. However, this is not bad because the colouring will extend to a total $\Lambda$-colouring of $G$ unless $\widehat{L}(x)$ and $\widehat{L}(y)$ are both equal to the set $\{a, b\}=\Lambda(w) \backslash \Lambda(w x)$, as given by (6). In contrast, pattern (i) is always bad. In checking Table 5 , it may help to observe that in every row of Table $4,|\Lambda(x) \cap \Lambda(x y)| \geqslant 3$ and $|\Lambda(x y) \cap \Lambda(y)| \geqslant 3$, and if $|\Lambda(x) \cap \Lambda(x y) \cap \Lambda(y)| \geqslant 3$ then $\Lambda(x y)$ is equal to $\Lambda(x)$ or $\Lambda(y)$. In each row of Table 5 where pattern (i) can arise, an appropriate condition is given which rules out all the bad colourings, so that any colouring satisfying that condition will extend to a total $\Lambda$-colouring of $G$.

Case 2: $\Lambda(w x)=\Lambda(w y)$ and $c \neq \lambda_{u}$ or $c \neq \lambda_{v}$, w.l.o.g. $c \neq \lambda_{u}$.

From Fig. 3(a), $\lambda_{u x}=\lambda_{v y}=d$, say, and $\Lambda(x y)=\{a, b, c, d\}$ and $\Lambda(x)=\left\{a, b, d, \lambda_{u}\right\}$, and so $\{c\}=\Lambda(x y) \backslash \Lambda(x)$. Now $\lambda_{u}, \lambda_{u x}, \lambda_{v y}, \lambda_{v}$ are uniquely determined by (6), and so any colouring satisfying an appropriate condition (condition $A$, for example) will extend to the whole of $G$, unless it gives rise to pattern (i). Since $\Lambda(x) \neq \Lambda(x y)$ but $\Lambda(x), \Lambda(x y), \Lambda(y)$ all have $a, b, d$ in common, the only row of Table 4 that could correspond is row 3 with $c$ and $d$ interchanged, when $\lambda_{u}=e$ and $\lambda_{v}=c$, as given in the penultimate row of Table 5 .

Case 3: $\Lambda(w x)=\Lambda(w y)$ and $c=\lambda_{u}=\lambda_{v}$.

As in Case 2, $\lambda_{u x}=\lambda_{v y}=d$, say, and so $\Lambda(x)=\Lambda(x y)=\Lambda(y)=\{a, b, c, d\}$, putting us in row 1 of Table 4 . We cannot distinguish between $c$ and $d$, and so the bad colourings for pattern (ii) are $c, d, d, c$ and $d, c, c, d$, as in the last row of Table 5 .

In every case, there is one of the conditions in Table 1 such that, provided the colouring of $u, u x, v, v y$ satisfies that condition, it can be extended to a total $\Lambda$-colouring of $G$. This shows that $G$ is good for total 4-choosability, and it completes the proof of Lemma 8 .

Lemma 9. If a nontrivial sepachain $G_{1}$ is good for total 4-choosability, then so is the sepachain $G$ in Fig. 1(h).

Proof. Let $\Lambda$ be a 4-list-assignment to $G$, and let colours $\lambda_{u}, \lambda_{u x}, \lambda_{v y}, \lambda_{v}$ be assigned to $u, u x, v y, v$. For each uncoloured element $z$, let $\widehat{L}(z)$ denote the set of all colours that can 
now be used on $z$, and for $z \in\{s x, x, x y, y, y t\}$ choose $L(z) \subseteq \widehat{L}(z)$ such that

$$
|L(z)|= \begin{cases}2 & \text { if } z=x, x y \text { or } y \\ 3 & \text { if } z=s x \text { or } y t\end{cases}
$$

We wish to prove that, by imposing a suitable restriction on the colours $\lambda_{u}, \lambda_{u x}, \lambda_{v y}, \lambda_{v}$ if necessary, we can colour $s x, x, x y, y, y$ from these lists in such a way that this colouring can be extended to the whole of $G_{1}$. Up to left-right reflections, the lists $L(x), L(x y), L(y)$ fall into one of the patterns (i)-(xii) shown in Table 3. (The columns headed $w x$ and $w y$ are not now relevant and should be ignored.)

The proof is in two parts. In Part 1 we show that every colouring $\lambda_{u}, \lambda_{u x}, \lambda_{v y}, \lambda_{v}$ of $u, u x, v y, v$ extends to the whole of $G$ unless it gives rise to pattern (i) or (up to left-right reflection and relabelling of the colours) to one of the following six 'problem cases'; in Part 2 we examine these problem cases in more detail.

$$
\left.\begin{array}{c}
\widehat{L}(x)=\{a, c\}, \quad \widehat{L}(x y)=\{a, b\}, \quad \widehat{L}(y)=\{b, c\}, \\
\widehat{L}(s x)=\{a, c, d\}, \quad \widehat{L}(y t)=\{a, b, c\}, \\
G_{1} \text { has type } J^{\prime}(d, b, a, c) ;
\end{array}\right\}
$$

Note that more than one of these cases may arise for the same pair $(G, \Lambda)$, with different choices of $\lambda_{u}, \lambda_{u x}, \lambda_{v y}, \lambda_{v}$ and (therefore) different labellings of the colours. 
Part 1. In this part of the proof we show that if the colouring $\lambda_{u}, \lambda_{u x}, \lambda_{v y}, \lambda_{v}$ does not give rise to pattern (i) or to any of (7)-(12), then it extends to the whole of $G_{1}$. We consider four cases.

Case 1.1: The pattern is (ii) or (vi), and $L(s x)=L(y t)=\{a, b, c\}$.

These patterns permit the colourings

$$
\begin{aligned}
& \begin{array}{llllllllll}
s x & x & x y & y & y t & s x & x & x y & y & y t
\end{array}
\end{aligned}
$$

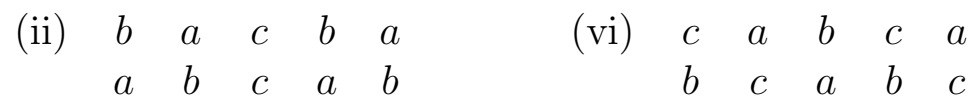

respectively. Rearranged in the appropriate order for comparison with Table 1 (applied to $G_{1}$-note that $\left.(x, s x, y t, y)=\left(u_{1}, u_{1} x_{1}, v_{1} y_{1}, v_{1}\right)\right)$, these become

$$
\begin{aligned}
& \begin{array}{llllllll}
x & s x & y t & y & x & s x & y t & y
\end{array}
\end{aligned}
$$

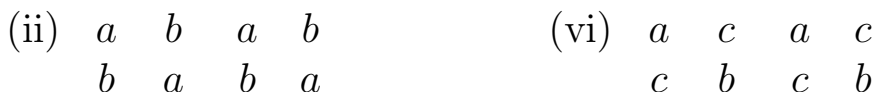

respectively. A comparison with Table 1 shows that if $G_{1}$ has any type other than $A$ or $B$ or $B^{\prime}$, then at least one colouring in each set will extend to the whole of $G_{1}$. If any of the sets $\widehat{L}(s x), \widehat{L}(x), \widehat{L}(x y), \widehat{L}(y), \widehat{L}(y t)$ contains any colour other than $a, b, c$ (and the hypotheses of Case 1.1 hold), then it is easy to see that there is a colouring that will extend even if $G_{1}$ has type $A$ or $B$ or $B^{\prime}$; that is, the colouring $\lambda_{u}, \lambda_{u x}, \lambda_{v y}, \lambda_{v}$ extends to the whole of $G_{1}$ unless (8) holds. (The last part of (8) is not relevant at this point.)

Case 1.2: The pattern is (vii) or (viii), and $L(s x)=\{a, b, c\}$ and $L(y t)=\{a, b, d\}$.

The four possible colourings are

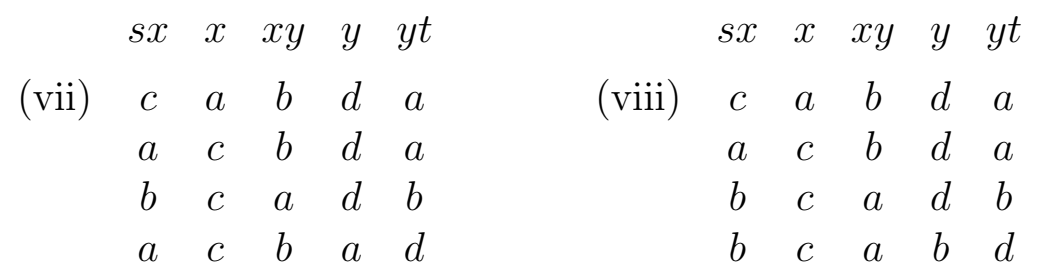

respectively. Rearranged, these become

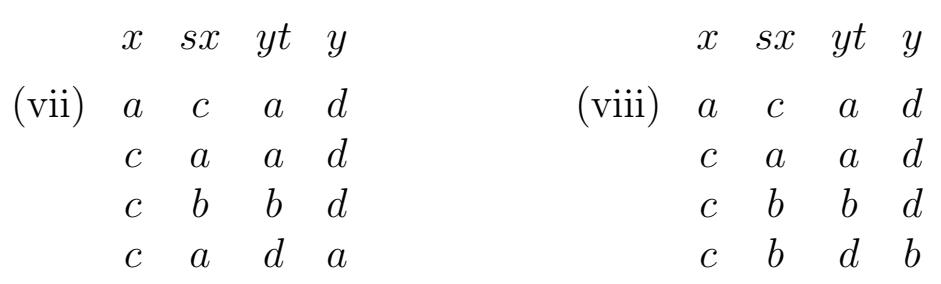

and it is easy to see that at least one of them must extend to the whole of $G_{1}$ : the two colourings in which $s x$ and $y t$ have the same colour must extend unless $G_{1}$ has type $A, C$, 


\begin{tabular}{|c|c|c|c|c|}
\hline Condition & $\begin{array}{r}\text { Forbid } \\
\text { of }\end{array}$ & $\begin{array}{l}\text { len colo } \\
\text { s } x, x, y \text {, }\end{array}$ & arings & $\begin{array}{c}\text { Bad pair of } \\
\text { colourings }\end{array}$ \\
\hline$B(a, b)$ & $\nu \mu \nu \mu$ & $b a \cdot a$ & & $(b / \nu) a \nu a$ \\
\hline$C(a, b)$ & $\nu a \nu b$ & $\xi a \cdot \xi$ & & $(b / \nu) a \nu b$ \\
\hline$E(a, b, c)$ & $\nu a \nu b$ & $c a \cdot b$ & & $(c / \nu) a \nu b$ \\
\hline$G^{\prime}(a, b, c)$ & $a \mu b \mu$ & $a c \cdot c$ & $b c b c$ & $(a / b) c b c$ \\
\hline$J^{\prime}(a, b, c, d)$ & $a \mu b \mu$ & $a c \cdot c$ & $d c d c$ & $(a / d) c d c$ \\
\hline$L^{\prime}(a, b, c, d)$ & $a \mu b \mu$ & $b c b c$ & $d c d c$ & $(a / b) c b c$ \\
\hline$M^{\prime}(a, b, c, d)$ & $a \mu b \mu$ & $d c b c$ & & $(a / d) c b c$ \\
\hline$O(a, b, c)$ & $b a a c$ & $\xi a a \xi$ & & $(b / c) a a c$ \\
\hline$S^{\prime}(a, b, c, d)$ & $b \cdot c$ & $d a d c$ & & $(b / d) a d c$ \\
\hline$U^{\prime}(a, b, c, d)$ & $b a a c$ & daac & & $(b / d) a a c$ \\
\hline$Z(a, b, c, d)$ & $b a d c$ & $\xi a d \xi$ & & $(b / c) a d c$ \\
\hline $\bar{A}^{\prime}(a, b, c, d)$ & $b a \cdot c$ & $d \cdot d c$ & & $(b / d) a d c$ \\
\hline $\bar{D}^{\prime}(a, b, c, d, e)$ & $b a d c$ & $e \cdot d c$ & & $(b / e) a d c$ \\
\hline
\end{tabular}

Table 6

$C^{\prime}$ or $Z$, in which case at least one of the other colourings in each column must extend. This completes Case 1.2.

Case 1.3: Cases 1.1 and 1.2 do not apply, and the pattern is not (iii) (or (i), of course).

Up to permutations of the letters, patterns (ii), (vi), (vii) and (viii) are left-right symmetric. In view of this and the results of Cases 1.1 and 1.2, we may assume that if the pattern is one of these four then $L(s x) \neq\{a, b, c\}$. Then in all cases it is possible to choose a colouring for $x, x y, y, y t$ that can be extended to $s x$ in at least two different ways. (For example, in patterns (iv), (v) and (ix)-(xii), $L(s x)$ cannot contain all of $a, b, c, d$, and whichever one it omits, at least one of the colourings $a, c$ and $b, d$ for $x, x y$ will extend to $s x$ in two different ways, regardless of the colours we give to $y$ and $y t$.) A study of Table 1 (or, more briefly, Table 2) shows that at least one of these two colourings must extend to the whole of $G_{1}$, unless $G_{1}$ has one of the types listed in Table 6 . Here we have listed the restrictions on the colourings in the order $s x, x, y, y t$, which is convenient for our present purpose, although it does not correspond to the order in Table 1; note however that the letters used in Table 6 agree with those in Table 1 and not necessarily with those in Table 3.

In Table 6, types $O$ and $U^{\prime}$ are irrelevant and are included only for completeness, since $x$ and $y$ are adjacent in $G$ and so cannot be given the same colour. Types $Z$ and $\bar{D}^{\prime}$ are also easily dismissed, since in each of these cases every forbidden colouring for $G_{1}$ has a fixed pair of colours on some pair of adjacent elements ( $a, d$ on $x, y$ for type $Z$, and $d, c$ on $y, y t$ for type $\bar{D}^{\prime}$ ), but every pattern allows for a colouring avoiding any such fixed pair of colours. 
If $G_{1}$ has type $C$ or $E$, then every forbidden colouring has the same colour $a$ on $x$, and every pattern allows for a colouring avoiding any such fixed colour on $x$ (except for pattern (iii), which we are not considering in Case 1.3). If $G_{1}$ has type $S^{\prime}$ or $\bar{A}^{\prime}$, then every forbidden colouring has a fixed colour $c$ on $y$ t, and every pattern allows for a colouring avoiding any such fixed colour on $y t$ except for pattern (iv). However, if the pattern is (iv) then we can choose the colour of $y$ to be different from the colour that is called $d$ in Table 6 , to avoid the second forbidden colouring in $S^{\prime}$ or $\bar{A}^{\prime}$, and we can then choose the colour of $x$ so that $s x$ can be given a different colour from the colour called $b$ in Table 6 , to avoid the first forbidden colouring in each case; then the resulting colouring can be extended to the whole of $G_{1}$.

Finally, the forbidden colourings for conditions $B, G^{\prime}, J^{\prime}, L^{\prime}$ and $M^{\prime}$ all require $x$ and $y t$ to have the same colour, and this can be avoided unless the lists are as in pattern (ii) or (vi) with $L(y t)=\{a, b, c\}$. However, by assumption in Case 1.3 there is a colour $d \in L(s x) \backslash\{a, b, c\}$, so that these patterns permit the colourings

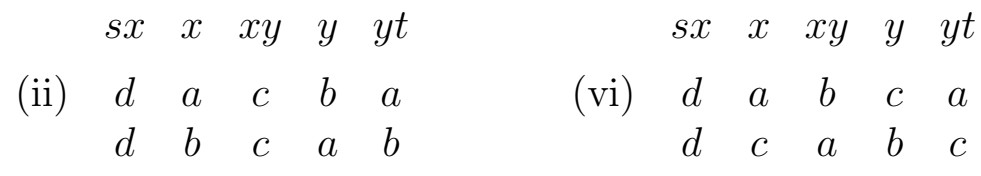

respectively. Of the stated conditions, the only ones that can rule out both colourings for the same pattern are $G^{\prime}(d, b, a)$ and $J^{\prime}(d, b, a$, ?), with pattern (vi). However, $|L(s x)| \geqslant 3$, and so pattern (vi) permits another colouring for $s x, x, x y, y, y$, either $c^{\prime}, a, b, c, a$ (where $c^{\prime}$ could equal $c$ ) or $b^{\prime}, c, a, b, c$ (where $b^{\prime}$ could equal $b$ ). Condition $G^{\prime}(d, b, a)$ cannot rule out either of these, and $J^{\prime}(d, b, a, ?)$ cannot rule out the second; but it can rule out the first if (and only if) it occurs as $J^{\prime}(d, b, a, c)$ and $c^{\prime}=c$. This problem arises only if the lists are exactly as in (7); in all other cases one of the colourings can be extended to the whole of $G_{1}$. This completes the discussion of Case 1.3.

Case 1.4: The pattern is (iii).

Since we have already dealt with every other pattern, we may assume that $c \in \widehat{L}(x) \subseteq$ $\{a, b, c\}$ and $\widehat{L}(x y)=\widehat{L}(y)=\{a, b\}$. Suppose first that $\widehat{L}(s x)=\widehat{L}(y t)=\{a, b, c\}$. Then we have the colourings on the left below, rearranged on the right:

$\begin{array}{lllllllll}s x & x & x y & y & y t & x & s x & y t & y \\ a & c & b & a & c & c & a & c & a \\ b & c & a & b & c & c & b & c & b\end{array}$

Comparison with Table 1 shows that the conditions that forbid both of these colourings are precisely those listed in (8), together with those obtained from the last six of these by interchanging $a$ and $b$. However, $a$ and $b$ are otherwise equivalent in (8), and so, by interchanging them if necessary, we may assume that at least one of the above colourings extends to the whole of $G_{1}$ unless (8) holds. 
Now suppose that $\widehat{L}(y t) \neq \widehat{L}(s x)=\{a, b, c\}$, so that there is a colour $d \in \widehat{L}(y t) \backslash$ $\{a, b, c\}$. Then we have the colourings on the left below, rearranged on the right:

\begin{tabular}{|c|c|c|c|c|}
\hline$s x$ & $x \quad x y$ & $y \quad y t$ & $x$ & $s x$ \\
\hline$a$ & $b$ & $d$ & $c$ & $a$ \\
\hline$b$ & $a$ & $b$ & $c$ & $b$ \\
\hline
\end{tabular}

Comparison with Table 1 shows that the conditions that forbid both of these colourings are precisely those listed in (9), together with those obtained from the last three of these by interchanging $a$ and $b$. Since $a$ and $b$ are otherwise equivalent in (9), we may assume that if $\widehat{L}(y t)=\{a, b, d\}$ then at least one of these colourings extends to the whole of $G_{1}$ unless (9) holds. If however $\widehat{L}(y t) \neq\{a, b, d\}$, then there is a different colour, either $c$ or a new colour $e$, available for $y t$. If $c \in \widehat{L}(y t)$ then we can give $x, s x, y t, y$ the colours in (13) as well as those in (14), and least one of these colourings must extend to the whole of $G_{1}$ since the types listed in (8) are disjoint from those in (9). If $e \in \widehat{L}(y t)$ then we can give $x, s x, y t, y$ the colours $c, a, e, a$ or $c, b, e, b$ as well as those in (14), and it is easy to see from Table 1 that none of the conditions listed in (9) can rule out both of these new colourings.

We have now dealt with the possibility that $\widehat{L}(s x)=\{a, b, c\}$, and so we may assume there is a colour $d \in \widehat{L}(s x) \backslash\{a, b, c\}$. Thus at least one colouring of $x, x y, y, y t$ can be extended to $s x$ in two different ways, and (as in Case 1.3) at least one of these extensions will extend to the whole of $G_{1}$ unless $G_{1}$ has one of the types listed in Table 6 . As in Case 1.3, types $O$ and $U^{\prime}$ are irrelevant, and types $Z$ and $\bar{D}^{\prime}$ are easily dismissed. We must consider the remaining nine conditions.

Since $a$ and $b$ are interchangeable in pattern (iii), we may assume that there exists a colour $a^{\prime} \in \widehat{L}(s x) \backslash\{b, c, d\}$, and then we have the colourings shown in the three columns below, depending on whether $\widehat{L}(y t) \backslash\{a, b\}$ contains $c, d$ or a new colour $e$.

$\begin{array}{ccccc}s x & x & x y & y & y t \\ a^{\prime} & c & b & a & c \\ d & c & b & a & c \\ d & c & a & b & c\end{array}$

$\begin{array}{ccccc}s x & x & x y & y & y t \\ a^{\prime} & c & b & a & d \\ d & c & b & a & d \\ d & c & a & b & d\end{array}$

$\begin{array}{ccccc}s x & x & x y & y & y t \\ a^{\prime} & c & b & a & e \\ d & c & b & a & e \\ d & c & a & b & e\end{array}$

It is easy to see that none of the nine remaining conditions in Table 6 can rule out all three colourings in any column if $a \neq a^{\prime}$, and so we may assume that $\widehat{L}(s x) \subseteq\{a, b, c, d\}$ and $a=a^{\prime}$. Then only conditions $B(c, d), G^{\prime}(d, a, c)$ and $J^{\prime}(d, ?, c, a)$ can rule out all three colourings in the first column, only condition $C(c, d)$ can rule out all three in the second column, and only $E(c, e, d), S^{\prime}(c, d, e, a)$ and $\bar{A}^{\prime}(c, d, e, a)$ can rule out all three in the third column. It immediately follows that if $|\widehat{L}(y t) \backslash\{a, b\}| \geqslant 2$, so that all the colourings in two different columns are possible, or else there are two different choices for $e$ in the third column, then no condition can rule out all these colourings; thus we may assume that $\widehat{L}(y t)=\{a, b, c\},\{a, b, d\}$ or $\{a, b, e\}$, for the three columns, respectively. Moreover, if $b \in \widehat{L}(s x)$ then conditions $G^{\prime}(d, a, c)$ and $J^{\prime}(d, ?, c, a)$ cannot rule out the colouring 


$\begin{array}{cccccc}\text { Eqn } & \Lambda(s x) & \Lambda(x) & \Lambda(x y) & \Lambda(y) & \Lambda(y t) \\ (7) & a c d \lambda_{u x} & a c \lambda_{u} \lambda_{u x} & a b \lambda_{u x} \lambda_{v y} & b c \lambda_{v y} \lambda_{v} & a b c \lambda_{v y} \\ (8) & a b c \lambda_{u x} & a b c \lambda_{u} \lambda_{u x} & a b c \lambda_{u x} \lambda_{v y} & a b c \lambda_{v y} \lambda_{v} & a b c \lambda_{v y} \\ (9) & a b c \lambda_{u x} & a b \underline{c} \lambda_{u} \lambda_{u x} & a b \lambda_{u x} \lambda_{v y} & a b \lambda_{v y} \lambda_{v} & a b d \lambda_{v y} \\ (10) & \underline{a} b c \underline{d} \lambda_{u x} & a b \underline{c} \lambda_{u} \lambda_{u x} & a b \lambda_{u x} \lambda_{v y} & a b \lambda_{v y} \lambda_{v} & a b c \lambda_{v y} \\ (11) & \underline{a} b c \underline{d} \lambda_{u x} & a b \underline{c} \lambda_{u} \lambda_{u x} & a b \lambda_{u x} \lambda_{v y} & a b \lambda_{v y} \lambda_{v} & a b d \lambda_{v y} \\ (12) & \underline{a} b c \underline{d} \lambda_{u x} & a b \underline{c} \lambda_{u} \lambda_{u x} & a b \lambda_{u x} \lambda_{v y} & a b \lambda_{v y} \lambda_{v} & a b e \lambda_{v y}\end{array}$

Table 7

$b, c, a, b, c$ for $s x, x, x y, y, y t$, and conditions $S^{\prime}(c, d, e, a)$ and $\bar{A}^{\prime}(c, d, e, a)$ cannot rule out the colouring $b, c, a, b, e$; thus in these four cases we may assume that $\widehat{L}(s x)=\{a, c, d\}$. In the other three cases, $a$ and $b$ are interchangeable, and so there is no loss of generality in assuming that $a \in \widehat{L}(s x)$. In other words, at least one colouring will extend unless (10), (11) or (12) holds.

Part 2. We have now shown that every colouring $\lambda_{u}, \lambda_{u x}, \lambda_{v y}, \lambda_{v}$ of $u, u x, v y, v$ extends to the whole of $G$ unless it gives rise to pattern (i) or (up to left-right reflection) to one of (7)(12). We must now examine (7)-(12), which we do in four cases. The lists corresponding to (7)-(12) are given in Table 7; when five colours are given for a particular list, that means that the list contains four of the five colours, including any that are underlined.

We work mainly with the lists, referring to the type of $G_{1}$ only when it seems that more than one of (7)-(12) may arise for the same pair $(G, \Lambda)$. Note that $(7)-(12)$ have been specified up to left-right reflection, and so it appears possible that, for a given pair $(G, \Lambda)$, one choice of $\lambda_{u}, \lambda_{u x}, \lambda_{v y}, \lambda_{v}$ may put us into one of these problem cases as shown, and another choice may put us into a different case in reflected form. This can happen only if the type of $G_{1}$ is, up to reflection, one of those listed in Case 2.1 below, with first two parameters $d, c$ (not $c, d$ ), and some choice of $\lambda_{u}, \lambda_{u x}, \lambda_{v y}, \lambda_{v}$ puts us into the reflected form of (9), which we denote by $\left(9^{\prime}\right)$, and a different choice puts us into the unreflected form of (7), (8) or (10). In Case 2.1 we show that in fact this cannot happen, and we also deal completely with $(7)$.

Case 2.1: The type of $G_{1}$ is $G^{\prime}, H^{\prime}, J^{\prime}, K^{\prime}, L^{\prime}$ or $N^{\prime}$, and the lists permit (7) or $\left(9^{\prime}\right)$ to hold.

In this case, if (8) holds, then $\widehat{L}(x y)=\widehat{L}(y)=\{a, b\}$; (9) can arise only in its reflected form $\left(9^{\prime}\right)$; if (10) holds then $\widehat{L}(s x)=\{a, c, d\}$; and (11) and (12) cannot arise. Thus, for this case only, we have the following modified form of Table 7 . 


$\begin{array}{cccccc}\text { Eqn } & \Lambda(s x) & \Lambda(x) & \Lambda(x y) & \Lambda(y) & \Lambda(y t) \\ (7) & a c d \lambda_{u x} & a c \lambda_{u} \lambda_{u x} & a b \lambda_{u x} \lambda_{v y} & b c \lambda_{v y} \lambda_{v} & a b c \lambda_{v y} \\ (8) & a b c \lambda_{u x} & a b c \lambda_{u} \lambda_{u x} & a b \lambda_{u x} \lambda_{v y} & a b \lambda_{v y} \lambda_{v} & a b c \lambda_{v y} \\ \left(9^{\prime}\right) & a b d \lambda_{u x} & a b \lambda_{u} \lambda_{u x} & a b \lambda_{u x} \lambda_{v y} & a b \underline{c} \lambda_{v y} \lambda_{v} & a b c \lambda_{v y} \\ (10) & a c d \lambda_{u x} & a b \underline{c} \lambda_{u} \lambda_{u x} & a b \lambda_{u x} \lambda_{v y} & a b \lambda_{v y} \lambda_{v} & a b c \lambda_{v y}\end{array}$

Table $7^{\prime}$

In Case 2.2 we will consider the possibility that the lists permit (8) only to arise; in Case 2.3 we will consider the possibility that $\left(9^{\prime}\right)$ or $(10)$ (but not both) can arise (although we will consider $\left(9^{\prime}\right)$ in its reflected form, i.e. (9), with the type of $G_{1}$ similarly reflected); and in Case 2.4 we will consider the possibility that (8) and (10) can both arise. In the present case, we will deal with all other possibilities. We will defer until last the discussion of the more complicated types $G^{\prime}$ and $J^{\prime}$.

Subcase 2.1.1: $G_{1}$ has type $H^{\prime}(p, q, r), K^{\prime}(p, q, r, \bar{s}), L^{\prime}(p, q, r, \bar{s})$ or $N^{\prime}(p, q, r, \bar{s}, \bar{t})$, and (8) and $\left(9^{\prime}\right)$ can both arise.

Then, in (8), $(a, b, c)$ is equal to $(r, q, p)$ if the type of $G_{1}$ is $H^{\prime}$, to $(\bar{s}, r, p)$ if it is $K^{\prime}$, to $(\bar{s}, q, r)$ if it is $L^{\prime}$, and to $(\bar{s}, \bar{t}, r)$ if it is $N^{\prime}$, while, in $\left(9^{\prime}\right),(d, c)=(p, q)$ in all cases. Thus the relevant lists from Table $7^{\prime}$ can be written as follows.

$\begin{array}{ccccccc}\text { Type } & \text { Eqn } & \Lambda(s x) & \Lambda(x) & \Lambda(x y) & \Lambda(y) & \Lambda(y t) \\ H^{\prime} & (8) & p q r \lambda_{u x}^{1} & p q r \lambda_{u}^{1} \lambda_{u x}^{1} & q r \lambda_{u x}^{1} \lambda_{v y}^{1} & q r \lambda_{v y}^{1} \lambda_{v}^{1} & p q r \lambda_{v y}^{1} \\ K^{\prime} & (8) & p r \bar{s} \lambda_{u x}^{1} & p r \bar{s} \lambda_{u}^{1} \lambda_{u x}^{1} & r \bar{s} \lambda_{u x}^{1} \lambda_{v y}^{1} & r \bar{s} \lambda_{v y}^{1} \lambda_{v}^{1} & p r \bar{s} \lambda_{v y}^{1} \\ L^{\prime} & (8) & q r \bar{s} \lambda_{u x}^{1} & q r \bar{s} \lambda_{u}^{1} \lambda_{u x}^{1} & q \bar{s} \lambda_{u x}^{1} \lambda_{v y}^{1} & q \bar{s} \lambda_{v y}^{1} \lambda_{v}^{1} & q r \bar{s} \lambda_{v y}^{1} \\ N^{\prime} & (8) & r \bar{s} \bar{t} \lambda_{u x}^{1} & r \bar{s} \bar{t} \lambda_{u}^{1} \lambda_{u x}^{1} & \bar{s} \bar{t} \lambda_{u x}^{1} \lambda_{v y}^{1} & \bar{s} \bar{t} \lambda_{v y}^{1} \lambda_{v}^{1} & r \bar{s} \bar{t} \lambda_{v y}^{1} \\ \text { All } & \left(9^{\prime}\right) & a^{2} b^{2} p \lambda_{u x}^{2} & a^{2} b^{2} \lambda_{u}^{2} \lambda_{u x}^{2} & a^{2} b^{2} \lambda_{u x}^{2} \lambda_{v y}^{2} & a^{2} b^{2} \underline{q} \lambda_{v y}^{2} \lambda_{v}^{2} & a^{2} b^{2} q \lambda_{v y}^{2}\end{array}$

Here every colour not occurring as a parameter in the type is given a superscript, since we do not know, for example, that the colour called $\lambda_{u x}$ in (8) is the same as the colour called $\lambda_{u x}$ in $\left(9^{\prime}\right)$.

Suppose first that $G_{1}$ has type $H^{\prime}$ or $K^{\prime}$. Note that $p, q, r, \bar{s}$ are distinct by definition, since $a, b, c, d$ represent distinct colours in Table 1 . We see from the entries for $\Lambda(y t)$ that $p \in\left\{a^{2}, b^{2}, \lambda_{v y}^{2}\right\}$, so that $p \in \Lambda(x y)$. Thus $p=\lambda_{u x}^{1}$ or $\lambda_{v y}^{1}$. But then either $\Lambda(s x)$ or $\Lambda(y t)$ does not contain four distinct colours. Since we are assuming in the present subcase that the type of $G_{1}$ is such that (8) and $\left(9^{\prime}\right)$ can both arise, this contradiction shows that $G_{1}$ does not have type $H^{\prime}$ or $K^{\prime}$.

If, however, $G_{1}$ has type $L^{\prime}$ or $N^{\prime}$, then we can get exactly the same contradiction by using $r$ instead of $p$. We see from the entries for $\Lambda(y t)$ that $r \in\left\{a^{2}, b^{2}, \lambda_{v y}^{2}\right\}$, so that $r \in \Lambda(x y)$. Thus $r=\lambda_{u x}^{1}$ or $\lambda_{v y}^{1}$. But then either $\Lambda(s x)$ or $\Lambda(y t)$ does not contain four distinct colours. This contradiction shows that Subcase 2.1.1 cannot arise.

Subcase 2.1.2: $G_{1}$ has type $G^{\prime}(p, q, r)$, and either (8) or (10) can arise together with $\left(9^{\prime}\right)$. Then $(p, q, r)$ is equal to $(b, a, c)$ in $(8)$ or $(d, a, c)$ in $(10)$, while $(p, q)=(d, c)$ in $\left(9^{\prime}\right)$. So the relevant lists from Table $7^{\prime}$ can be written as follows. 


$\begin{array}{cccccc}\text { Eqn } & \Lambda(s x) & \Lambda(x) & \Lambda(x y) & \Lambda(y) & \Lambda(y t) \\ (8) & p q r \lambda_{u x}^{1} & p q r \lambda_{u}^{1} \lambda_{u x}^{1} & p q \lambda_{u x}^{1} \lambda_{v y}^{1} & p q \lambda_{v y}^{1} \lambda_{v}^{1} & p q r \lambda_{v y}^{1} \\ \left(9^{\prime}\right) & a^{2} b^{2} p \lambda_{u x}^{2} & a^{2} b^{2} \lambda_{u}^{2} \lambda_{u x}^{2} & a^{2} b^{2} \lambda_{u x}^{2} \lambda_{v y}^{2} & a^{2} b^{2} q \lambda_{v y}^{2} \lambda_{v}^{2} & a^{2} b^{2} q \lambda_{v y}^{2} \\ (10) & p q r \lambda_{u x}^{3} & b^{3} q \underline{r} \lambda_{u}^{3} \lambda_{u x}^{3} & b^{3} q \lambda_{u x}^{3} \lambda_{v y}^{3} & b^{3} q \lambda_{v y}^{3} \lambda_{v}^{3} & b^{3} q r \lambda_{v y}^{3}\end{array}$

Whether it is (8) and $\left(9^{\prime}\right)$ that both hold, or (10) and $\left(9^{\prime}\right)$, we get a contradiction in exactly the same way as for types $L^{\prime}$ and $N^{\prime}$ in Subcase 2.1.1, using the fact that $r \in\left\{a^{2}, b^{2}, \lambda_{v y}^{2}\right\}$ but $r \notin \Lambda(x y)$. (Note that $r \neq b^{3}$ in (10).)

Subcase 2.1.3: $G_{1}$ has type $J^{\prime}(p, q, r, \bar{s})$.

Then $(p, r, \bar{s})$ is equal to $(b, c, a)$ in $(8)$ or $(d, c, a)$ in $(10)$, and $(p, q, r, \bar{s})=(d, b, a, c)$ in $(7)$, while $(p, q)=(d, c)$ in $\left(9^{\prime}\right)$. So the relevant lists from Table $7^{\prime}$ can be written as follows.

$\begin{array}{cccccc}\text { Eqn } & \Lambda(s x) & \Lambda(x) & \Lambda(x y) & \Lambda(y) & \Lambda(y t) \\ (7) & p r \bar{s} \lambda_{u x}^{0} & r \bar{s} \lambda_{u}^{0} \lambda_{u x}^{0} & q r \lambda_{u x}^{0} \lambda_{v y}^{0} & q \bar{s} \lambda_{v y}^{0} \lambda_{v}^{0} & q r \bar{s} \lambda_{v y}^{0} \\ (8) & p r \bar{s} \lambda_{u x}^{1} & p r \bar{s} \lambda_{u}^{1} \lambda_{u x}^{1} & p \bar{s} \lambda_{u x}^{1} \lambda_{v y}^{1} & p \bar{s} \lambda_{v y}^{1} \lambda_{v}^{1} & p r \bar{s} \lambda_{v y}^{1} \\ \left(9^{\prime}\right) & a^{2} b^{2} p \lambda_{u x}^{2} & a^{2} b^{2} \lambda_{u}^{2} \lambda_{u x}^{2} & a^{2} b^{2} \lambda_{u x}^{2} \lambda_{v y}^{2} & a^{2} b^{2} q \lambda_{v y}^{2} \lambda_{v}^{2} & a^{2} b^{2} q \lambda_{v y}^{2} \\ (10) & p r \bar{s} \lambda_{u x}^{3} & b^{3} \underline{r} \bar{s} \lambda_{u}^{3} \lambda_{u x}^{3} & b^{3} \bar{s} \lambda_{u x}^{3} \lambda_{v y}^{3} & b^{3} \bar{s} \lambda_{v y}^{3} \lambda_{v}^{3} & b^{3} r \bar{s} \lambda_{v y}^{3}\end{array}$

If $(7)$ and $\left(9^{\prime}\right)$ hold, we get a contradiction in the same way as before, by deducing that $\bar{s} \in\left\{a^{2}, b^{2}, \lambda_{v y}^{2}\right\}$ but $\bar{s} \notin \Lambda(x y)$. If (8) or (10) holds, along with $\left(9^{\prime}\right)$, then we get a contradiction because $r \in\left\{a^{2}, b^{2}, \lambda_{v y}^{2}\right\}$ but $r \notin \Lambda(x y)$. If (8) or (10) holds as well as (7), then $\bar{s} \in \Lambda(x y)$ and so $\bar{s}=\lambda_{u x}^{0}$ or $\lambda_{v y}^{0}$, which is impossible from the lists for $\Lambda(s x)$ and $\Lambda(y t)$. The remaining possibilities for $(8),\left(9^{\prime}\right)$ and (10) to hold will be considered in Cases $2.2-2.4$.

To complete Case 2.1 we consider the possibility that (7) holds. Note that $p, q, r, \bar{s}$ are known from the type of $G_{1}$, and so $\lambda_{u}^{0}, \lambda_{u x}^{0}, \lambda_{v y}^{0}, \lambda_{v}^{0}$ are determined by the lists, since $\left\{\lambda_{u x}^{0}\right\}=\Lambda(s x) \backslash\{p, r, \bar{s}\},\left\{\lambda_{u}^{0}\right\}=\Lambda(x) \backslash\left\{r, \bar{s}, \lambda_{u x}^{0}\right\}$, etc. Thus there is exactly one assignment of colours $\lambda_{u}^{0}, \lambda_{u x}^{0}, \lambda_{v y}^{0}, \lambda_{v}^{0}$ to $u, u x, v y, v$ that can cause (7) to hold, and it can be avoided by imposing a suitable condition on $G$, e.g. condition $D\left(\lambda_{u x}^{0}, \lambda_{v y}^{0}\right)$. However, we must consider also the possibility that pattern (i) can arise, as in Table 4 . Note that $\bar{s}$ is in $\Lambda(x)$ and $\Lambda(y)$ but not $\Lambda(x y)$, and the only row of Table 4 in which there is such an element is row 4. As in every row of Table $4,|\Lambda(x) \cap \Lambda(x y)| \geqslant 3$, and so to match this row $\lambda_{u}^{0}$ must equal $q$ or $\lambda_{v y}^{0}$. Similarly $|\Lambda(x y) \cap \Lambda(y)| \geqslant 3$, and so $\lambda_{v}^{0}$ must equal $r$ or $\lambda_{u x}^{0}$. Rewriting $q, r, \bar{s}, \lambda_{u x}^{0}, \lambda_{v y}^{0}$ as $b, a, c, d$, e, we have the four possibilities in the following table. In every case there is a condition that avoids all the bad colourings, and this completes the discussion of Subcase 2.1.3 and hence of Case 2.1.

Colourings

\begin{tabular}{|c|c|c|c|c|c|}
\hline$\Lambda(x)$ & $\Lambda(x y)$ & $\Lambda(y)$ & $\begin{array}{c}\lambda_{u} \lambda_{u x} \\
\text { bac } \\
(7)\end{array}$ & $\begin{array}{l}\lambda_{v y} \lambda_{v} \\
\text { for } \\
\quad \text { (i) }\end{array}$ & $\begin{array}{l}\text { Avoided by } \\
\text { condition }\end{array}$ \\
\hline$a c d b$ & $a b d e$ & bce $a$ & $b d e a$ & $c d e c$ & $D(d, e)$ \\
\hline$a c d e$ & $a b d e$ & bce $a$ & edea & $c d b c$ & $X(c, d, b, e, ?)$ \\
\hline$a c d b$ & $a b d e$ & bced & $b d e d$ & caec & $X^{\prime}(c, a, e, d, ?)$ \\
\hline$a c d e$ & $a b d e$ & $b c e d$ & eded & $c a b c$ & $Y(c, a, b, e, d)$ \\
\hline
\end{tabular}


Case 2.2: The lists, and the type of $G_{1}$, permit (8) to hold, but none of (9)-(12).

We see from Table 7 (not Table $7^{\prime}$ ) that if $\Lambda(s x)=\Lambda(y t)$ then (8) arises only when $\lambda_{u x}=\lambda_{v y}$, and it can be avoided by imposing condition $A$ on $G$. If $\Lambda(s x) \neq \Lambda(y t)$ then (8) arises only when $\left\{\lambda_{u x}\right\}=\Lambda(s x) \backslash \Lambda(y t)$ and $\left\{\lambda_{v y}\right\}=\Lambda(y t) \backslash \Lambda(s x)$, and it can be avoided by imposing condition $D\left(\lambda_{u x}, \lambda_{v y}\right)$ on $G$. However, we must check the possibility that pattern (i) arises, as in Table 4. A complete list of all the possibilities as in Table 5 would be too long, because $\Lambda(x), \Lambda(x y)$ and $\Lambda(y)$ are not uniquely determined by the values of $\lambda_{u x}$ and $\lambda_{v y}$ that give rise to (8); and so we need a different approach. We consider two subcases.

Subcase 2.2.1: $\Lambda(s x)=\Lambda(y t)=\{a, b, c, d\}$, say, and (8) arises when $\lambda_{u x}=\lambda_{v y}=d$.

Then $\Lambda(x y)=\{a, b, c, d\}, \Lambda(x) \subseteq\left\{a, b, c, d, \lambda_{u}\right\}$ and $\Lambda(y) \subseteq\left\{a, b, c, d, \lambda_{v}\right\}$. For the purpose of tabulating possibilities for the sets $\Lambda(x)$ and $\Lambda(y)$, it is convenient to assume that $\lambda_{u} \in \Lambda(x)$ and $\lambda_{v} \in \Lambda(y)$, since if $\lambda_{u} \notin \Lambda(x)$ then the set $\Lambda(x)$ is the same as it would be if $\lambda_{u}=a$, say. So, using $a$ and $b$ to denote 'anything in $\{a, b, c, d\}$ ', the essentially different possibilities for $\left(\lambda_{u}, \lambda_{v}\right)$ are $(a, b),(e, b),(e, e)$ and $(e, f)$, where distinct letters denote distinct colours as usual, since $(a, e)$ is equivalent to $(e, b)$ under left-right reflection. If $\lambda_{u}=e$ then we may assume that $\Lambda(x)=\{a, b, c, e\}$, since (as far as the sets are concerned) $a, b, c, d$ are all interchangeable; and then, if $\lambda_{v}=e$ or $f, \Lambda(y)$ is either $\left\{a, b, c, \lambda_{v}\right\}$ or $\left\{a, b, d, \lambda_{v}\right\}$. The possibilities for the lists $\Lambda(x), \Lambda(x y), \Lambda(y)$ are given in the top six rows of Table 8 , together with the colourings $\lambda_{u}, \lambda_{u x}, \lambda_{v y}, \lambda_{v}$ that will cause the lists $\widehat{L}(x), \widehat{L}(x y), \widehat{L}(y)$ to be as in (8) or (i) (up to colour permutations), and the conditions from Table 1 that will prevent this from happening.

Subcase 2.2.2: $\Lambda(s x) \neq \Lambda(y t)$, say $\Lambda(s x)=\{a, b, c, d\}$ and $\Lambda(y t)=\{a, b, c, e\}$, and (8) can arise if (and only if) $\lambda_{u x}=d$ and $\lambda_{v y}=e$.

Then $\Lambda(x y) \subset\{a, b, c, d, e\}, \Lambda(x) \subseteq\left\{a, b, c, d, \lambda_{u}\right\}$ and $\Lambda(y) \subseteq\left\{a, b, c, e, \lambda_{v}\right\}$. Note that each of $a, b, c$ must occur in at least one of these sets, since otherwise we could not have (8) except as pattern (i). As in Subcase 2.1.1, we will assume that $\lambda_{u} \in \Lambda(x)$ and $\lambda_{v} \in \Lambda(y)$.

Suppose first that $d$ does not occur in any of these sets, so that $\Lambda(x y)=\{a, b, c, e\}$, $\Lambda(x)=\left\{a, b, c, \lambda_{u}\right\}, \lambda_{u} \notin\{a, b, c, d\}$, and $\lambda_{v} \neq d$. The possibilities for $\left(\lambda_{u}, \lambda_{v}\right)$ now (using $b$ to denote 'anything in $\{a, b, c, e\}$ ') are $(e, b),(e, f),(f, b),(f, f)$ and $(f, g)$, and if $\lambda_{v} \neq b$ then we may assume that $\Lambda(y)$ is either $\left\{a, b, c, \lambda_{v}\right\}$ or $\left\{a, b, e, \lambda_{v}\right\}$, since (as far as the other sets are concerned) $a, b, c$ are all interchangeable. The possibilities are listed in the next eight rows of Table 8 , together with the colourings $\lambda_{u}, \lambda_{u x}, \lambda_{v y}, \lambda_{v}$ that will cause the lists $\widehat{L}(x), \widehat{L}(x y), \widehat{L}(y)$ to be as in (8) or (i) (up to colour permutations-but since $\Lambda(s x) \cap \Lambda(y t)=\{a, b, c\},(8)$ requires that $\lambda_{u x}=d, \lambda_{v y}=e$ and $\widehat{L}(x), \widehat{L}(x y), \widehat{L}(y) \subseteq$ $\{a, b, c\}$, and so the lists must be exactly as in (8)). In each case there is an appropriate condition that will prevent this from happening. In view of this, we may assume that $d$ occurs in at least one of the sets $\Lambda(x), \Lambda(x y), \Lambda(y)$, and similarly, by symmetry, that $e$ also occurs in at least one set. Thus each of $a, b, c, d, e$ occurs in at least one set.

Suppose now that there is another colour $f$ in one of the sets, w.l.o.g. $\lambda_{v}=f(f \notin$ $\{a, b, c, d, e\})$. Then we cannot have pattern (i) except as row 5 of Table 4 . Note that 


\begin{tabular}{|c|c|c|c|c|c|c|c|}
\hline$\left(\lambda_{u}, \lambda_{v}\right)$ & $\Lambda(x)$ & $\Lambda(x y)$ & $\Lambda(y)$ & $\begin{array}{l}\text { Colourings } \\
\lambda_{u} \lambda_{u x} \lambda_{v y} \lambda_{v} \\
\text { bad for }(8)\end{array}$ & $\begin{array}{l}\text { Row of } \\
\text { Table } 4\end{array}$ & $\begin{array}{l}\text { Colourings } \\
\lambda_{u} \lambda_{u x} \lambda_{v y} \lambda_{v} \\
\text { bad for }(\mathrm{i})\end{array}$ & $\begin{array}{l}\text { Avoided by } \\
\text { condition }\end{array}$ \\
\hline$(a, b)$ & $a b c d$ & $a b c d$ & $a b c d$ & $\cdot \xi \xi \cdot$ & 1 & $\mu \nu \mu \nu$ & A \\
\hline$(e, b)$ & $a b c e$ & $a b c d$ & $a b c d$ & $e \xi \xi$. & 3 & $e \nu d \nu$ & $C(e, d)$ \\
\hline \multirow[t]{2}{*}{$(e, e)$} & $a b c e$ & $a b c d$ & $a b c e$ & $e \xi \xi e$ & - & & \\
\hline & $a b c e$ & $a b c d$ & $a b d e$ & $e \xi \xi e$ & 4 & $e c d e$ & $O(e, c, d)$ \\
\hline \multirow[t]{2}{*}{$(e, f)$} & $a b c e$ & $a b c d$ & $a b c f$ & $e \xi \xi f$ & - & & \\
\hline & $a b c e$ & $a b c d$ & $a b d f$ & $e \xi \xi f$ & 5 & $e c d f$ & $Z(e, c, d, f)$ \\
\hline$(e, b)$ & $a b c e$ & $a b c e$ & $a b c e$ & $e d e$. & 1 & $\mu \nu \mu \nu$ & $B(e, d)$ \\
\hline \multirow[t]{2}{*}{$(e, f)$} & $a b c e$ & $a b c e$ & $a b c f$ & edef & 2 & $\mu e \mu f$ & $I^{\prime}(e, f, d)$ \\
\hline & $a b c e$ & $a b c e$ & $a b e f$ & $e d e f$ & 2 & $\mu c \mu f$ & $M^{\prime}(c, f, e, d)$ \\
\hline$(f, b)$ & $a b c f$ & $a b c e$ & $a b c e$ & $f d e$. & 3 & $f \nu e \nu$ & $E(f, e, d)$ \\
\hline \multirow{2}{*}{$(f, f)$} & $a b c f$ & $a b c e$ & $a b c f$ & $f d e f$ & - & & \\
\hline & $a b c f$ & $a b c e$ & $a b e f$ & $f d e f$ & 4 & $f c e f$ & $U^{\prime}(f, c, e, d)$ \\
\hline \multirow[t]{2}{*}{$(f, g)$} & $a b c f$ & $a b c e$ & $a b c g$ & $f d e g$ & - & & \\
\hline & $a b c f$ & $a b c e$ & $a b e g$ & $f d e g$ & 5 & $f c e g$ & $\bar{D}^{\prime}(f, c, e, g, d)$ \\
\hline \multirow[t]{2}{*}{$(a, f)$} & $a b c d$ & $a b c e$ & $a b e f$ & $\cdot d e f$ & 5 & $d c e f$ & $\bar{B}^{\prime}(d, c, e, f)$ \\
\hline & $a b c d$ & $a b d e$ & $a b e f$ & $\cdot d e f$ & 5 & $c d e f$ & $D(d, e)$ \\
\hline \multirow[t]{11}{*}{$(e, f)$} & $a b d e$ & $a b c d$ & $a b c f$ & edef & 5 & $e d c f$ & $E^{\prime}(d, f, c)$ \\
\hline & $a b d e$ & $a b c e$ & $a b c f$ & edef & 5 & $d e c f$ & $\bar{C}^{\prime}(d, e, c, f)$ \\
\hline & $a b d e$ & $a b c e$ & ace $f$ & edef & 5 & $d b c f$ & $\bar{E}^{\prime}(d, b, c, f, e)$ \\
\hline & $a b d e$ & $a c d e$ & acef & $e d e f$ & 5 & $b d c f$ & $E^{\prime}(d, f, c)$ \\
\hline & $a b c d$ & $a b c d$ & $a b c e$ & - $d e$ & 2 & $\mu d \mu e$ & $D^{\prime}(d, e)$ \\
\hline & $a b c d$ & $a b c d$ & $a b d e$ & $\cdot d e d$ & 2 & $\mu c \mu e$ & $F^{\prime}(c, e, d)$ \\
\hline & $a b c e$ & $a b c e$ & $a b c d$ & eded & 2 & $\mu e \mu d$ & $H^{\prime}(e, d, ?)$ \\
\hline & $a b c e$ & $a b c e$ & $a b d e$ & eded & 2 & $\mu c \mu d$ & $L^{\prime}(c, d, e)$ \\
\hline & $a b d e$ & $a b d e$ & $a b c d$ & eded & 2 & $\mu e \mu c$ & $H^{\prime}(e, c, d)$ \\
\hline & $a b d e$ & $a b d e$ & $a b c e$ & $e d e$ & 2 & $\mu d \mu c$ & $J^{\prime}(d, c, e, ?)$ \\
\hline & $a b d e$ & $a b d e$ & acde & eded & 2 & $\mu b \mu c$ & $N^{\prime}(b, c, e, d, ?)$ \\
\hline
\end{tabular}

Table 8 


\begin{tabular}{|c|c|c|c|}
\hline $\begin{array}{l}\text { Common } \\
\text { colours }\end{array}$ & $\begin{array}{l}\text { Colourings } \\
\lambda_{u} \lambda_{u x} \lambda_{v y} \lambda_{v} \\
\text { bad for }(\mathrm{i})\end{array}$ & $\begin{array}{l}\text { Colourings } \\
\lambda_{u} \lambda_{u x} \lambda_{v y} \lambda_{v} \\
\text { bad for }(8)\end{array}$ & $\begin{array}{c}\text { Avoided by } \\
\text { condition }\end{array}$ \\
\hline \multirow[t]{6}{*}{$a, b$} & $c d e c$ & - $d e$. & $D(d, e)$ \\
\hline & decd & eded & $Q^{\prime}(d, e, c)$ \\
\hline & $e c d e$ & eded & $Q(e, c, d)$ \\
\hline & $c e d c$ & eded & $R(c, e, d)$ \\
\hline & $e d c e$ & $e d e$ & $P(e, d, c)$ \\
\hline & $d c e d$ & $\cdot d e d$ & $P^{\prime}(d, c, e)$ \\
\hline \multirow[t]{3}{*}{$c, d$} & $a b e a$ & $\cdot d e d$ & $\bar{A}^{\prime}(a, b, e, d)$ \\
\hline & $b e a b$ & eded & $W(b, e, a, d)$ \\
\hline & $e a b e$ & eded & $V(e, a, b, d)$ \\
\hline$\{d, e\}$ & $a b c a$ & eded & $Y(a, b, c, e, d)$ \\
\hline
\end{tabular}

Table 9

in this row there is no colour that belongs to $\Lambda(x)$ and $\Lambda(y)$ but not to $\Lambda(x y)$, and so we do not need to consider the possibility that $\lambda_{u}=\lambda_{v}=f$; and also there is no colour that belongs to $\Lambda(x y)$ but not to $\Lambda(x)$ or $\Lambda(y)$, and so, since $d$ is in at least one set, we may assume that $d \in \Lambda(x)$. Since $a, b, c$ are all interchangeable, and $\lambda_{u} \neq \lambda_{u x}=d$, we may assume that $\left(\lambda_{u}, \lambda_{v}\right)=(a, f)$ or $(e, f)$, and in the latter case $\Lambda(x)=\{a, b, d, e\}$ and $\Lambda(y)=\{a, b, c, f\}$ or $\{a, c, e, f\}$, since $|\Lambda(x) \cap \Lambda(y)|=2$. The possibilities are listed in the next six rows of Table 8 .

Finally we assume that all three sets are subsets of $\{a, b, c, d, e\}$, and we consider rows 1, 2 and 4 of Table 4 in turn (row 3 being essentially the same as row 2). For row 1, we have already considered the case when all three sets equal $\{a, b, c, e\}$. The only essentially different case is when they all equal $\{a, b, d, e\}$; but in this case (8) cannot arise except as (i) (when $u, u x, v y, y$ have colours $e, d, e, d$ ), and so there is nothing more to consider. For row 2, the three essentially different possibilities for the first two (equal) sets are $\{a, b, c, d\},\{a, b, c, e\}$ and $\{a, b, d, e\}$; the first two each give two possibilities for $\Lambda(y)$ (since $a, b, c$ are interchangeable), and the last gives three possibilities (since only $a, b$ are interchangeable), as listed in the last seven rows of Table 8.

In row 4 of Table 4 , there are two colours that belong to all three sets, and every other colour belongs to two sets. The three essentially different possibilities for the two colours in all three sets are $\{a, b\}$ (which is equivalent to $\{a, c\}$ and $\{b, c\}$ ), $\{c, d\}$ (which is equivalent to $\{a, d\},\{b, d\},\{a, e\},\{b, e\}$ and $\{c, e\})$, and $\{d, e\}$; and for each of these the other three colours can be used in 3 ! different ways. However, in the second case the other three colours are $a, b, e$, and since $a$ and $b$ are interchangeable there are only three essentially different ways of ordering these; and in the last case the other three colours are $a, b, c$, and all six orderings of these are essentially equivalent. The possibilities are listed in Table 9 . The three sets are not given in the table, but can easily be reconstructed: if 
the common colours are $p, q$ and the bad colouring for (i) is $i j k i$, then $\Lambda(x)=\{p, q, i, j\}$, $\Lambda(x y)=\{p, q, j, k\}$ and $\Lambda(y)=\{p, q, k, i\}$, and the first element of the bad colouring for (8) is $e$ if $e \in \Lambda(x)$ and - otherwise, and the last element is $d$ if $d \in \Lambda(y)$ and * otherwise. In every case there is an appropriate condition that rules out all the bad colourings, and this completes the discussion of Case 2.2.

Case 2.3: The lists, and the type of $G_{1}$, permit at least one of (9)-(12) to hold, but not (8).

We start by proving the following claim.

Claim. For a given pair $(G, \Lambda)$, there is at most one choice of colour for ux, and at most two choices of colour for vy, that can give rise to any of (9)-(12). Moreover, if any of (9)-(12) holds when vy is given colour c, then there is no other choice of colour for vy that can give rise to any of (9)-(12).

Proof. We consider two subcases.

Subcase 2.3.1: $G_{1}$ has type $E(c, p, q), S^{\prime}(c, q, p, ?)$ or $\bar{A}^{\prime}(c, q, p, ?)$, and both (9) and (12) can hold, with different labellings of the colours $(p=d$ in $(9)$, and $(p, q)=(e, d)$ in $(12))$.

From Table 7 we see that the lists are as follows.

$\begin{array}{cccccc}\text { Eqn } & \Lambda(s x) & \Lambda(x) & \Lambda(x y) & \Lambda(y) & \Lambda(y t) \\ (9) & a^{1} b^{1} c \lambda_{u x}^{1} & a^{1} b^{1} \underline{c} \lambda_{u}^{1} \lambda_{u x}^{1} & a^{1} b^{1} \lambda_{u x}^{1} \lambda_{v y}^{1} & a^{1} b^{1} \lambda_{v y}^{1} \lambda_{v}^{1} & a^{1} b^{1} p \lambda_{v y}^{1} \\ (12) & \underline{a}^{2} b^{2} c \underline{q} \lambda_{u x}^{2} & a^{2} b^{2} \underline{c} \lambda_{u}^{2} \lambda_{u x}^{2} & a^{2} b^{2} \lambda_{u x}^{2} \lambda_{v y}^{2} & a^{2} b^{2} \lambda_{v y}^{2} \lambda_{v}^{2} & a^{2} b^{2} p \lambda_{v y}^{2}\end{array}$

Note that $a, b, c, d$ are distinct colours in (9) and $a, b, c, d, e$ are distinct colours in (12), and so $a^{1}, b^{1}, c, p$ are distinct and $a^{2}, b^{2}, c, q, p$ are distinct. From the lists for $\Lambda(y t)$ we see that $\left\{a^{1}, b^{1}, \lambda_{v y}^{1}\right\}=\left\{a^{2}, b^{2}, \lambda_{v y}^{2}\right\}$, so that, from $\Lambda(x y), \lambda_{u x}^{1}=\lambda_{u x}^{2},=\lambda_{u x}$, say; thus there is only one choice of colour for $u x$ that can cause (9) or (12) to hold. Now, $\lambda_{u x} \neq a^{2}$ or $q$, since $a, d \in \widehat{L}(s x)$ in (12), and $c \neq a^{2}$ or $q$; thus $\left\{a^{1}, b^{1}\right\}=\left\{a^{2}, q\right\}$. Since $a^{1}$ and $b^{1}$ are interchangeable in (9), we may suppose w.l.o.g. that $a^{1}=a^{2}$ and $b^{1}=q$. (Note that, in order to achieve this, we do not need to interchange the colours themselves, but only their names.) From $\Lambda(x y)$ it now follows that $\left\{b^{1}, \lambda_{v y}^{1}\right\}=\left\{b^{2}, \lambda_{v y}^{2}\right\}$, so that there are at most two choices for the colour of $v y$ (namely, the two elements of this set) that can give rise to (9) or (12). Finally, if $\lambda_{v y}^{1}=c$ or $\lambda_{v y}^{2}=c$ then $c \in \Lambda(y t)$, but $c \notin \widehat{L}(y t)$ in (9) or (12) and so $\lambda_{v y}^{1}=\lambda_{v y}^{2}=c$; thus in this case there is only one choice of colour for $v y$ that can give rise to (9) or (12). This proves the Claim in Subcase 2.3.1.

Subcase 2.3.2: $(G, \Lambda)$ and the type of $G_{1}$ are such that (9) and (12) cannot both arise.

If none of (9)-(12) can arise then there is nothing to prove; so assume that at least one can arise. If more than one can arise then $G_{1}$ must have type $C(c, d)$, and it is (9) and (11) that can arise. Note however that $c$ and $d$ have the same meaning in (9) as in (11). This differs from Subcase 2.3.1, where $d$ has different meanings (i.e., it represents different parameters in the type of $\left.G_{1}\right)$ in $(9)$ and $(12)$.

So consider a labelling of the colours that agrees with one of (9)-(12). Then $c$ and $d$, and $e$ if (12) holds, are given to us in the type of $G_{1}$. It is now clear from Table 7 
that from this knowledge and the lists we can uniquely determine the set $\left\{a, b, \lambda_{v y}\right\}$ as $\Lambda(y t) \backslash\{p\}$, where $p=c, d$ or $e$ as appropriate. Hence we can determine $\lambda_{u x}$ as the unique colour in $\Lambda(x y) \backslash X$, where $X:=\left\{a, b, \lambda_{v y}\right\}=\Lambda(y t) \backslash\{p\}$.

Let $Y:=\Lambda(s x) \backslash\left\{c, d, \lambda_{u x}\right\}$. Then $Y=\{a\}$ or $\{a, b\}$, and we know which, since these sets have different cardinality. But $\left\{\lambda_{v y}\right\}=X \backslash\{a, b\}$, and so if $|Y|=2$ then $\lambda_{v y}$ is uniquely determined. If $|Y|=1$ then $X \backslash Y=\left\{b, \lambda_{v y}\right\}$, and there may be two different choices of colour for $v y$ (namely, the two colours in $X \backslash Y$ ) that can give rise to (9)-(12). If however $c \in X$, then necessarily $\lambda_{v y}=c$, and so in this case there is only one choice for the colour of $v y$ that can give rise to any of (9)-(12). This completes the proof of the Claim.

If $G_{1}$ has type $B$ or $G^{\prime}$ or $J^{\prime}$, then it is possible that recolouring $v y$ with colour $b$ will move us from (10) to (8); but we will consider this possibility in Case 2.4, since it is ruled out by the hypotheses of Case 2.3. We will now examine the one or two possible bad colourings for (9)-(12), at the same time as we consider the possibility that (i) arises, as in Table 4.

For convenience, let us relabel $d$ as $d^{*}$ and $e$ (if it exists) as $e^{*}$, and relabel $\lambda_{u x}$ as $d$. (It is irrelevant whether or not $d$ or $e$ (introduced below) is equal to $d^{*}$ or $e^{*}$, since we will have no further use for the sets $\Lambda(s x)$ and $\Lambda(y t)$.) Then, from Table $7, \Lambda(x y)=\left\{a, b, d, \lambda_{v y}\right\}$, $\Lambda(y)=\left\{a, b, \lambda_{v y}, \lambda_{v}\right\}$, and $\lambda_{u}, c \in \Lambda(x) \subseteq\left\{a, b, c, d, \lambda_{u}\right\}$. (As in Case 2.2, for the purpose of listing the sets, we may assume that $\lambda_{u} \in \Lambda(x)$.) The possibilities for $\lambda_{v y}$ are that it equals $c$ or a new colour $e \notin\{a, b, c, d\}$. The possibilities are listed in Table 10, with $\lambda_{v y}=c$ in the top 11 lines and $\lambda_{v y}=e$ in the remainder. Note that $\lambda_{v} \notin\left\{a, b, \lambda_{v y}\right\}$, since otherwise $|\Lambda(y)| \leqslant 3$. So, using $a$ to denote 'anything in $\{a, b, c, d\}$ ', the essentially different possibilities for $\left(\lambda_{u}, \lambda_{v}\right)$ are $(a, c),(a, d),(a, f),\left(\lambda_{v y}, c\right),\left(\lambda_{v y}, d\right),\left(\lambda_{v y}, f\right),(f, c)$, $(f, d),(f, f)$ and $(f, g)$. However, if $\lambda_{v y}=c$ then we can rule out the five pairs in which $\lambda_{u}=\lambda_{v y}$ or $\lambda_{v}=c$, since the former is then equivalent to $\lambda_{u}=a$ and the latter is not then possible. In Table 10, the entries in the second of the two columns headed 'bad for (9)-(12)' assume that there is a choice of two bad colours (b and $e$ ) for $v y$; if there is only one bad colour then these second entries simply disappear. Note that there is only one bad colour for $v y$, and so there is only one colouring that is bad for (9)-(12), if $\lambda_{v y}=c$, or if $\lambda_{v y}=e$ and $\{b, c, f\} \subset \Lambda(x)$ (since it does not matter if $v y$ is given colour $b$, so that $\widehat{L}(x y)=\widehat{L}(y)=\{a, e\}$, if $\widehat{L}(x)$ contains more than one colour not in $\{a, e\})$. In every case there is an appropriate condition that rules out all the bad colourings, and this completes the discussion of Case 2.3.

Case 2.4: The lists, and the type of $G_{1}$, permit (8), and at least one of (9)-(12), to hold.

Then $G_{1}$ must have type $B$ or $G^{\prime}$ or $J^{\prime}$, and it must be (8) and (10) that can both hold. Assume that the colours are labelled as in (10). Then $c$ and $d$ are given to us in the type of $G_{1}$. As in Subcase 2.3.2, from the row for (10) in Table 7 we see that $\lambda_{u x}$ is the unique colour in $\Lambda(x y) \backslash X$, where $X:=\Lambda(y t) \backslash\{c\}$; and if $Y:=\Lambda(s x) \backslash\left\{c, d, \lambda_{u x}\right\}$ then $Y=\{a\}$ or $\{a, b\}$ and $\lambda_{v y} \in X \backslash Y$. Thus if $b \in \Lambda(s x)$ then the colours of $u x$ and $v y$ that can cause (10) to hold are uniquely determined, while if $b \notin \Lambda(s x)$ then the colour of $u x$ is uniquely determined and there are at most two different possible colours for $v y$ that can 


\begin{tabular}{|c|c|c|c|c|c|c|c|c|}
\hline \multirow{4}{*}{$\begin{array}{c}\left(\lambda_{u}, \lambda_{v}\right) \\
\\
(a, d) \\
(a, f) \\
(f, d)\end{array}$} & \multirow{2}{*}{$\begin{array}{l}\Lambda(x) \\
a b c d\end{array}$} & \multirow{2}{*}{$\begin{array}{l}\Lambda(x y) \\
a b c d\end{array}$} & $\Lambda(y)$ & \multicolumn{2}{|c|}{$\begin{array}{c}\text { Colourings } \\
\lambda_{u} \lambda_{u x} \lambda_{v y} \lambda_{v} \\
\text { bad for } \\
(9)-(12)\end{array}$} & $\begin{array}{l}\text { Row of } \\
\text { Table } 4\end{array}$ & $\begin{array}{c}\text { Colourings } \\
\lambda_{u} \lambda_{u x} \lambda_{v y} \lambda_{v} \\
\text { bad for } \\
\text { (i) }\end{array}$ & $\begin{array}{l}\text { Avoided by } \\
\text { condition }\end{array}$ \\
\hline & & & $a b c d$ & $\cdot d c d$ & & 1 & $\mu \nu \mu \nu$ & $B^{\prime}(c, d)$ \\
\hline & $a b c d$ & $a b c d$ & $a b c f$ & $\cdot d c f$ & & 2 & $\mu d \mu f$ & $E^{\prime}(d, f, c)$ \\
\hline & $a b c f$ & $a b c d$ & $a b c d$ & $f d c d$ & & 3 & $f \nu d \nu$ & $I(f, d, c)$ \\
\hline \multirow{4}{*}{$(f, f)$} & $a c d f$ & $a b c d$ & $a b c d$ & $f d c d$ & & 3 & $f \nu b \nu$ & $M(f, b, d, c)$ \\
\hline & $b c d f$ & $a b c d$ & $a b c d$ & $f d c d$ & & 3 & $f \nu a \nu$ & $M(f, a, d, c)$ \\
\hline & $a b c f$ & $a b c d$ & $a b c f$ & $f d c f$ & & - & & \\
\hline & $a c d f$ & $a b c d$ & $a b c f$ & $f d c f$ & & 4 & $f d b f$ & $U(f, d, b, c)$ \\
\hline \multirow{4}{*}{$(f, g)$} & $b c d f$ & $a b c d$ & $a b c f$ & $f d c f$ & & 4 & $f d a f$ & $U(f, d, a, c)$ \\
\hline & $a b c f$ & $a b c d$ & $a b c g$ & $f d c g$ & & - & & \\
\hline & $a c d f$ & $a b c d$ & $a b c g$ & $f d c g$ & & 5 & $f d b g$ & $D(f, d, b, g, c)$ \\
\hline & $b c d f$ & $a b c d$ & $a b c g$ & $f d c g$ & & 5 & $f d a g$ & $\bar{D}(f, d, a, g, c)$ \\
\hline$(a, c)$ & $a b c d$ & $a b d e$ & $a b e c$ & $\cdot d e c$ & $b d b c$ & 4 & $c d e c$ & $\bar{A}(b, d, e, c)$ \\
\hline$(a, d)$ & $a b c d$ & $a b d e$ & abed & - ded & $b d b d$ & 3 & $c \nu e \nu$ & $J(c, e, d, b)$ \\
\hline$(a, f)$ & $a b c d$ & $a b d e$ & $a b e f$ & $\cdot d e f$ & $b d b f$ & 5 & cdef & $\bar{A}(b, d, e, f)$ \\
\hline \multirow[t]{3}{*}{$(e, c)$} & $a b c e$ & $a b d e$ & $a b e c$ & $e d e c$ & $b d b c$ & - & - & $E^{\prime}(d, c, b)$ \\
\hline & acde & $a b d e$ & $a b e c$ & edec & $b d b c$ & 4 & $c d b c$ & $E^{\prime}(d, c, b)$ \\
\hline & $b c d e$ & $a b d e$ & $a b e c$ & edec & $b d b c$ & 4 & $c d a c$ & $E^{\prime}(d, c, a)$ \\
\hline \multirow[t]{3}{*}{$(e, d)$} & $a b c e$ & $a b d e$ & abed & eded & $b d b d$ & 3 & $c \nu d \nu$ & $K(c, d, b, e)$ \\
\hline & acde & $a b d e$ & abed & eded & $b d b d$ & 3 & $c \nu b \nu$ & $J(c, b, d, e)$ \\
\hline & $b c d e$ & $a b d e$ & abed & eded & $b d b d$ & 3 & $c \nu a \nu$ & $N(c, a, d, b, e)$ \\
\hline \multirow[t]{3}{*}{$(e, f)$} & $a b c e$ & $a b d e$ & $a b e f$ & edef & $b d b f$ & - & - & $E^{\prime}(d, f, b)$ \\
\hline & acde & $a b d e$ & $a b e f$ & edef & $b d b f$ & 5 & $c d b f$ & $E^{\prime}(d, f, b)$ \\
\hline & $b c d e$ & $a b d e$ & $a b e f$ & edef & $b d b f$ & 5 & $c d a f$ & $E^{\prime}(d, f, a)$ \\
\hline \multirow[t]{3}{*}{$(f, c)$} & $a b c f$ & $a b d e$ & $a b e c$ & $f d e c$ & & - & & \\
\hline & $a c d f$ & $a b d e$ & $a b e c$ & $f d e c$ & $f d b c$ & - & - & $D(f, d, b, c, e)$ \\
\hline & $b c d f$ & $a b d e$ & $a b e c$ & $f d e c$ & & - & & \\
\hline \multirow{2}{*}{$(f, d)$} & $\begin{array}{l}a b c f \\
a c d f\end{array}$ & abde & abed & fded & $f d h d$ & - & & \\
\hline & $b c d f$ & $\begin{array}{l}\text { abde } \\
\text { abde }\end{array}$ & $\begin{array}{l}\text { abed } \\
\text { abed }\end{array}$ & $\begin{array}{l}\text { fded } \\
\text { fded }\end{array}$ & $J a 0 a$ & - & - & $M(f, b, d, e)$ \\
\hline \multirow[t]{3}{*}{$(f, f)$} & $a b c f$ & $a b d e$ & $a b e f$ & $f d e f$ & & - & & \\
\hline & $\operatorname{acd} f$ & $a b d e$ & $a b e f$ & fdef & $f d b f$ & - & - & $U(f, d, b, e)$ \\
\hline & $b c d f$ & $a b d e$ & $a b e f$ & $f d e f$ & & - & & \\
\hline \multirow[t]{3}{*}{$(f, g)$} & $a b c f$ & $a b d e$ & abeg & $f d e g$ & & - & & \\
\hline & $\begin{array}{l}a c d f \\
b c d f\end{array}$ & $\begin{array}{l}a b d e \\
a b d e\end{array}$ & $\begin{array}{l}\text { abeg } \\
\text { abeg }\end{array}$ & $\begin{array}{l}f d e g \\
\text { fdea }\end{array}$ & $f d b g$ & - & - & $D(f, d, b, g, e)$ \\
\hline & & wo & & & & - & & \\
\hline
\end{tabular}

Table 10 


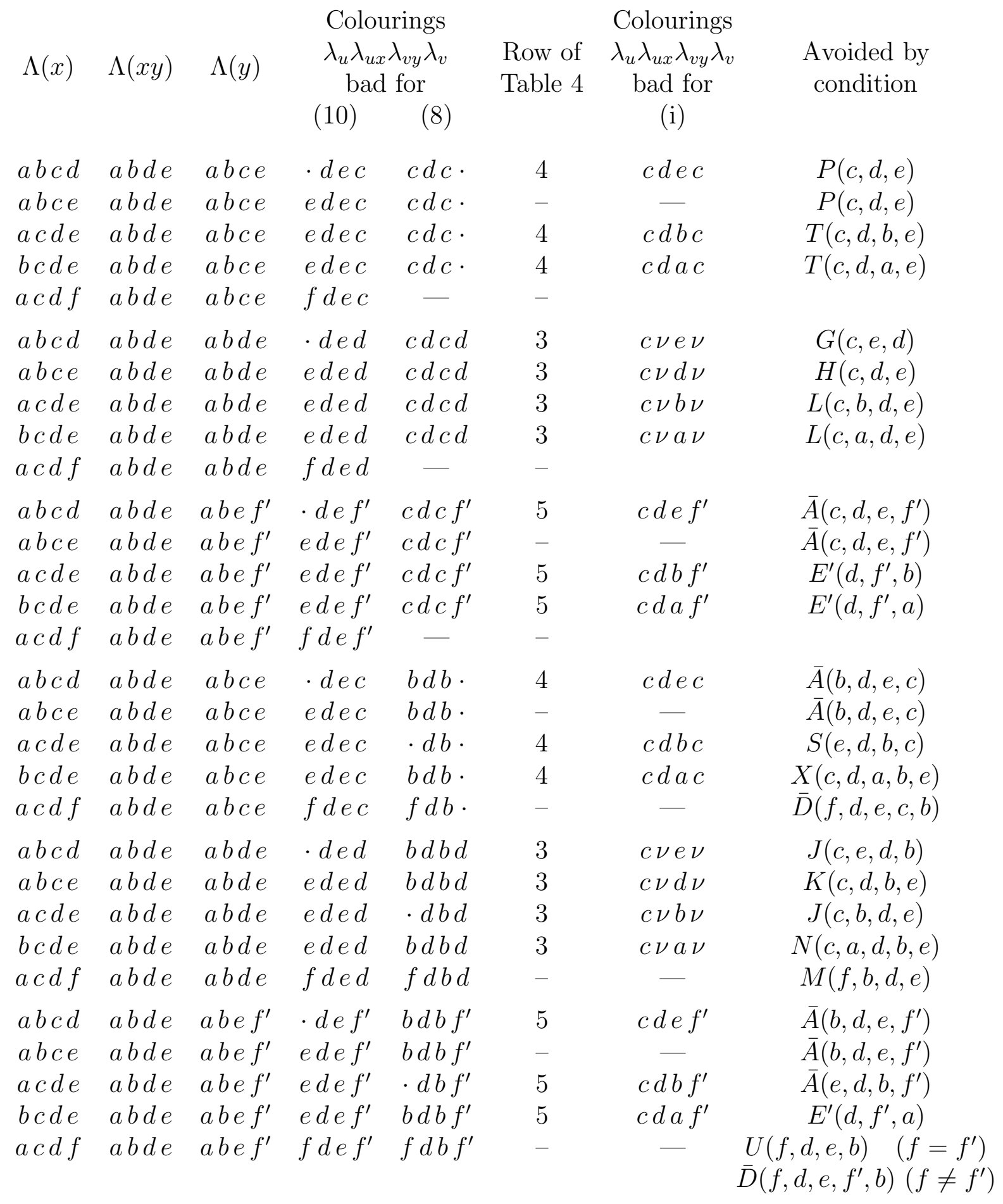

Table 11 
cause (10) to hold. Note that $\lambda_{u x} \neq a$ or $b$ in (10), since $a, b \in \widehat{L}(x y) \subset \Lambda(x y) \backslash\left\{\lambda_{u x}\right\}$; nor $c$, since $c \in \widehat{L}(x) \subseteq \Lambda(x) \backslash\left\{\lambda_{u x}\right\}$; nor $d$, since $d \in \widehat{L}(s x)=\Lambda(s x) \backslash\left\{\lambda_{u x}\right\}$. Thus $\lambda_{u x}$ is a new colour, say $e$. Note that $\lambda_{v y} \neq \lambda_{u x}$ and so $e \in \Lambda(x y) \backslash \Lambda(y t)$.

Now, with the colours still labelled as in (10), consider what is necessary in order for the lists in (8) to be possible as well (after relabelling of colours). (8) can arise only when $u x$ and $v y$ are coloured with colours in $\Lambda(s x)$ and $\Lambda(y t)$ respectively, after which $\widehat{L}(x y) \subseteq \widehat{L}(y t)$; but if $e \notin \Lambda(s x)$ then, whatever colours we choose for $u x$ and $v y, \widehat{L}(x y)$ will still contain $e$, which is not in $\widehat{L}(y t)$, so that (8) cannot arise. Thus we may assume that $e \in \Lambda(s x)$, so that $\{a, d, e\} \subset \Lambda(s x) \subset\{a, b, c, d, e\}$, and $b$ or $c \notin \Lambda(s x)$, from the row for (10) in Table 7 . From the row for (8) in Table 7 we see that (8) requires that $|\Lambda(s x) \cap \Lambda(y t)| \geqslant 3$, and the only way in which this is now possible in (10) is if $\lambda_{v y}=d$. For consistency with earlier cases, we now interchange the roles of $d$ and $e$, so that $\lambda_{u x}=d$, $\lambda_{v y}=e, c \in \Lambda(x) \subseteq\left\{a, b, c, d, \lambda_{u}\right\}, \Lambda(y)=\left\{a, b, e, \lambda_{v}\right\}$, and the lists become:

$\begin{array}{ccccc}\Lambda(s x) & \Lambda(x) & \Lambda(x y) & \Lambda(y) & \Lambda(y t) \\ \{a, b, d, e\} & \{a, b, c, d\} & \{a, b, d, e\} & \{a, b, c, e\} & \{a, b, c, e\} \\ \{a, c, d, e\} & \{a, b, c, e\} & & \{a, b, d, e\} & \\ & \{a, c, d, e\} & & \left\{a, b, e, f^{\prime}\right\} & \\ & \{b, c, d, e\} & & & \\ & {[\{a, b, c, f\}]} & & \\ & \{a, c, d, f\} & & \\ & {[\{b, c, d, f\}]} & & & \end{array}$

This is because the possibilities for $\lambda_{u}$ are that it is one of $a, b, c, d$, or $e$, or a new colour $f$; and $\lambda_{v}$, which cannot belong to $\{a, b, e\}$, is $c$, or $d$, or a new colour $f^{\prime}$; here distinct letters represent distinct colours, except that $f^{\prime}$ is allowed to be the same as $f$. However, not all the sets listed for $\Lambda(x)$ need to be considered, as we will see.

In the top half of Table 11 we consider all the possibilities with $\Lambda(s x)=\{a, b, d, e\}$, when $\Lambda(s x) \cap \Lambda(y t)=\{a, b, e\}$. Here (8) arises only when $\left\{\lambda_{u x}\right\}=\Lambda(s x) \backslash\{a, b, e\}=\{d\}$, $\left\{\lambda_{v y}\right\}=\Lambda(y t) \backslash\{a, b, e\}=\{c\}$, and $\Lambda(x) \backslash\left\{d, \lambda_{u}\right\} \subseteq\{a, b, e\}$; this implies that $\lambda_{u}=c$ (since $c \in \Lambda(x)$ always), and it is impossible if $\Lambda(x)=\{a, b, c, f\},\{a, c, d, f\}$ or $\{b, c, d, f\}$, so that we do not need to consider these possibilities. Note that $a$ and $b$ are otherwise interchangeable, and so if we consider $\Lambda(x)=\{a, c, d, e\}$ then we do not also need to consider $\Lambda(x)=\{b, c, d, e\}$, which will be obtained simply by interchanging $a$ and $b$. (However, the cases $\Lambda(x)=\{b, c, d, e\}$ and $\{a, c, d, f\}$ are included in the top half of Table 11, even though they are not needed there, for consistency with the bottom half, where they are needed.) In this case (10) arises only when $\lambda_{u x}=d$ and $\lambda_{v y}=e$, since (as we saw at the start of Case 2.4) if $b \in \Lambda(s x)$ then there is only one colour for $\lambda_{v y}$ that can cause (10) to arise.

In the bottom half of Table 11 we consider all the possibilities with $\Lambda(s x)=\{a, c, d, e\}$, when $\Lambda(s x) \cap \Lambda(y t)=\{a, c, e\}$. Here (8) arises only when $\left\{\lambda_{u x}\right\}=\Lambda(s x) \backslash\{a, c, e\}=\{d\}$, $\left\{\lambda_{v y}\right\}=\Lambda(y t) \backslash\{a, c, e\}=\{b\}$, and $\Lambda(x) \backslash\left\{d, \lambda_{u}\right\} \subseteq\{a, c, e\}$; this is impossible if $\Lambda(x)=\{a, b, c, f\}$ or $\{b, c, d, f\}$, since $\lambda_{u}$ cannot equal both $b$ and $f$, and so we do not 
need to consider these possibilities. In this case (10) arises only when $\lambda_{u x}=d$, but, as we saw at the start of Case 2.4, since $b \notin \Lambda(s x)$ we cannot determine from the lists whether

$\lambda_{v y}=e$ or $b$; however, choosing $\lambda_{v y}=b$ gives $\widehat{L}(s x)=\widehat{L}(y t)=\{a, c, e\}$ and so moves us out of (10) and into (8), and so it is covered automatically in Table 11.

In each case there is a condition that we can impose on $G$ in order to avoid all the bad colourings. This completes the discussion of Case 2.4, and with it the proof of Lemma 9.

\section{References}

[1] O. V. Borodin, A. V. Kostochka and D. R. Woodall, List edge and list total colourings of multigraphs, J. Combin. Theory Ser. B 71 (1997), 184-204.

[2] G. A. Dirac, A property of 4-chromatic graphs and some remarks on critical graphs, J. London Math. Soc. 27 (1952), 85-92.

[3] P. Erdős, A. L. Rubin and H. Taylor, Choosability in graphs, in: Proc. West Coast Conference on Combinatorics, Graph Theory and Computing, Arcata, 1979, Congr. Numer. 26 (1980), 125-157.

[4] A. J. W. Hilton and P. D. Johnson, The Hall number, the Hall index, and the total Hall number of a graph, Discrete Applied Math. 94 (1999), 227-245.

[5] T. J. Hetherington and D. R. Woodall, Edge and total choosability of nearouterplanar graphs, Electron. J. Combin. 13 (2006), \#R98, 7pp.

[6] M. Juvan, B. Mohar and R. Škrekovski, List total colourings of graphs, Combin. Probab. Comput. 7 (1998), 181-188.

[7] M. Juvan, B. Mohar and R. Thomas, List edge-colorings of series-parallel graphs, Electron. J. Combin. 6 (1999), \#R42, 6pp.

[8] A. V. Kostochka and D. R. Woodall, Choosability conjectures and multicircuits, Discrete Math. 240 (2001), 123-143.

[9] W. Wang and K.-W. Lih, Choosability, edge choosability, and total choosability of outerplane graphs, European J. Combin 22 (2001), 71-78.

[10] D. R. Woodall, List colourings of graphs, Surveys in Combinatorics, 2001 (ed. J. W. P. Hirschfeld), London Math. Soc. Lecture Note Series 288, Cambridge University Press, Cambridge, 2001, 269-301.

[11] D. R. Woodall, Some totally 4-choosable graphs, manuscript (2006).

[12] X. Zhou, Y. Matsuo and T. Nishizeki, List total colorings of series-parallel graphs, Computing and Combinatorics, Lecture Notes in Comput. Sci., 2697, Springer, Berlin, 2003, 172-181. 